This item was submitted to Loughborough's Research Repository by the author.

Items in Figshare are protected by copyright, with all rights reserved, unless otherwise indicated.

\title{
On the objective assessment and quantification of the transient-handling response of a vehicle
}

PLEASE CITE THE PUBLISHED VERSION

http://dx.doi.org/10.1080/00423110600862268

PUBLISHER

(c) Taylor \& Francis

VERSION

SMUR (Submitted Manuscript Under Review)

LICENCE

CC BY-NC-ND 4.0

\section{REPOSITORY RECORD}

Mavros, George. 2014. "On the Objective Assessment and Quantification of the Transient-handling Response of a Vehicle". figshare. https://hdl.handle.net/2134/14749. 
This item was submitted to Loughborough's Institutional Repository (https://dspace.lboro.ac.uk/) by the author and is made available under the following Creative Commons Licence conditions.

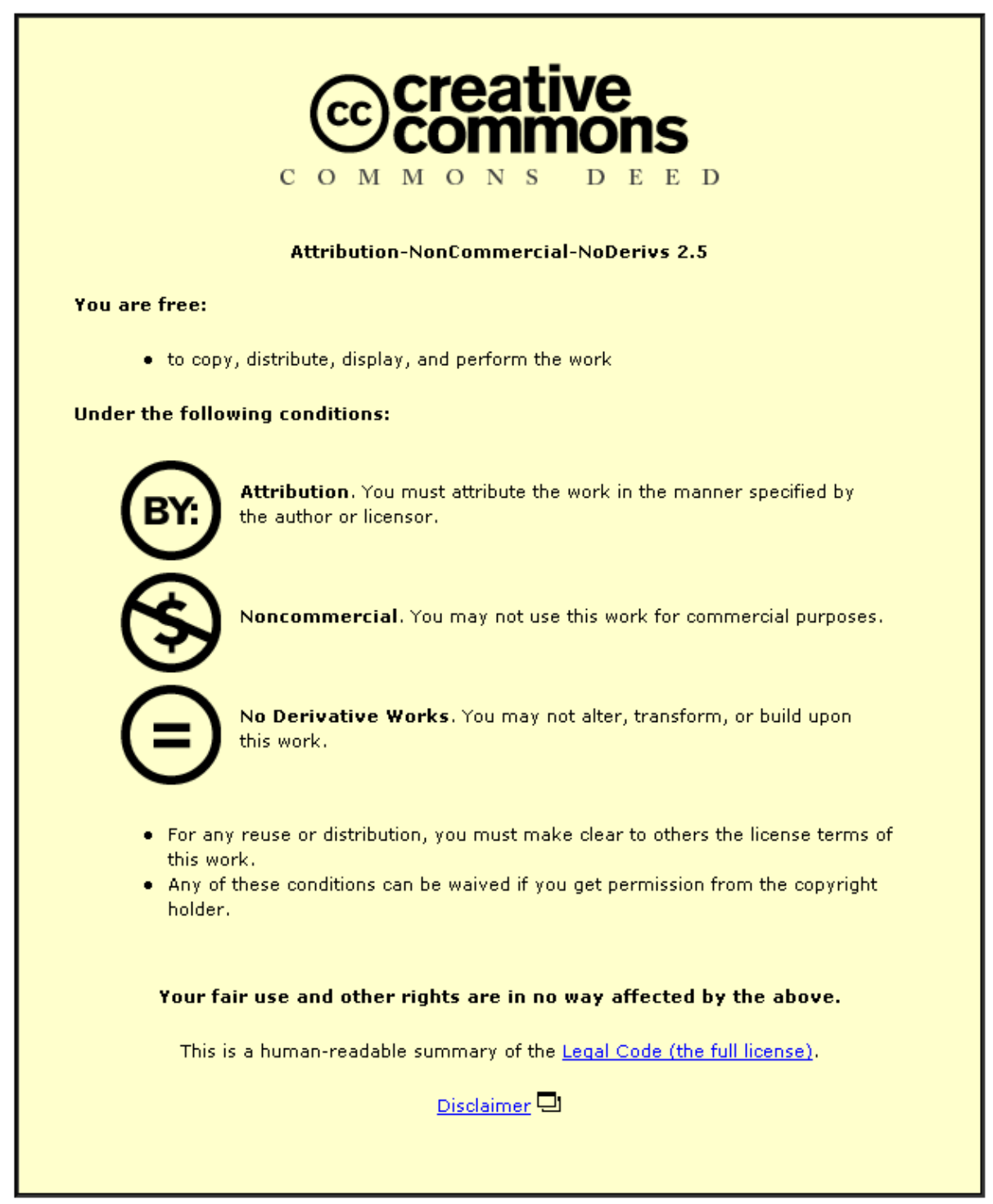

For the full text of this licence, please go to: http://creativecommons.org/licenses/by-nc-nd/2.5/ 
On the objective assessment and quantification of the transient handling response

of a vehicle

G. MAVROS*†

$\dagger$ School of Aeronautical and Automotive Engineering, Loughborough University,

LE113TU, UK

Contact Details:

Dr George Mavros

Aeronautical and Automotive Engineering

Loughborough University

Loughborough

Leicestershire

LE11 3TU

UK

Direct line: $+44(0) 1509227273$

Fax: +44(0)1509 227275

e-mail: g.mavros@lboro.ac.uk 


\begin{abstract}
The paper suggests a new methodology for the objective assessment and quantification of the response of a vehicle subjected to transient handling manoeuvres. For this purpose, a non-dimensional measure is defined, namely the normalised yaw impulse. This measure appears in two variations. In its general or dynamic form, it represents the difference between the yaw moment due to the front tyre forces and the yaw moment due to the rear tyre forces, divided by the sum of the aforementioned yaw moments. By employing a linear, 2 degree-of-freedom bicycle model it is shown that the general form of the normalised yaw impulse can be written as a function of the steer angle and the forward, lateral and yaw velocities of the vehicle. This form is referred to as the kinematic yaw impulse. It is demonstrated that the combined application of the dynamic and kinematic expressions of the yaw impulse not only facilitates the explicit assessment and quantification of the transient behaviour of a vehicle, but also reveals the influence of parameters such as the yaw moment of inertia, which traditionally leave the steady-state behaviour unaffected.
\end{abstract}

Keywords: Transient; Handling; Yaw moment; Tyre force; Measure; 


\section{$1 \quad$ Introduction}

The objective assessment of the handling performance is an important task in the design and testing process of every road vehicle, as it determines the levels of active safety and the responsiveness of the vehicle to the driver's commands. The handling behaviour is investigated either under steady-state or transient operating conditions. In both cases, the employment of the linear 2 degree-of-freedom bicycle model has provided researchers with invaluable insight. According to the definition proposed by SAE [1], a vehicle operates in steady-state when its response has reached a constant value which remains unchanged over an arbitrarily long time, or when its response has reached a periodic state which remains unchanged over an arbitrarily long time.

The most common and interesting case of constant response is that of a vehicle negotiating a turn of constant radius of curvature, while maintaining constant forward speed. The simplifications permitted by the linear bicycle model have lead to the definition of the well established under-steer coefficient (see for example the analysis in [2]). As a result, the steady state behaviour is explicitly defined, based on the sign of the under-steer coefficient. It is shown in [3-5] that the definition of the under-steer coefficient deteriorates when non-linearities are taken on-board. For such cases, Pacejka [3-5] proposes the use of the handling diagram which consists of the normalised centripetal acceleration, plotted against the difference between rear and front slip angles, while the vehicle negotiates a turn of constant radius. In this case, a vehicle is explicitly characterised based on the slope of the normalised acceleration curve. A very similar approach for the assessment of steady-state handling is employed by Lukowski et al [6]. 
The equations of motion of the linear bicycle model can be solved in order to provide the steady-state response to a sinusoidal steering input [2]. Using the Laplace transformation, the transfer function of the yaw rate with respect to the steer angle is easily derived and the corresponding bode graph plotted [2], providing a clear picture of the bandwidth of the vehicle's response [2]. Allen et al [7] have also employed bode plots for the assessment of the frequency response of a vehicle under combined cornering/braking conditions. In general, the frequency response (calculated numerically or experimentally for non-linear models or real vehicles, respectively) provides a rather informative image of a vehicle's handling qualities and can be also related to the transient response of the system, as is well known from basic dynamics and control theory [8]. For example, the inverse of a specified bandwidth frequency can be used as an equivalent time constant for the vehicle's yaw rate response to a step-steer input [7]. Furthermore, the existence of a resonance frequency indicates a system with sub-critical damping, which is related to under-steer [2], as opposed to over-steering vehicles which are characterised by an equivalent damping ratio greater than one [2]. Nevertheless, the general characterisation of a vehicle based on its frequency response should be attempted with caution. For instance, an observed resonance frequency which indicates under-steer, might be related to the roll frequency of the vehicle in conjunction with a suspension set-up which dictates severe lateral weight transfer at the front end only. Certainly, this condition would not apply on any manoeuvre and thus the overall characterisation of the vehicle proves to be a difficult mission.

The frequency response of a vehicle has already been related to its transient response to a step steer manoeuvre. According to the definition proposed by SAE [1], a vehicle operates in transient-state when the motion responses, the external forces relative to the 
vehicle, or the control positions are changing with time. The step steer input represents the most common transient handling test due to its mathematical simplicity and the insight it offers in the vehicle's overall behaviour. Once again, the linear bicycle model allows the analytical calculation of the transient time response. Before proceeding with the actual step-steer manoeuvre, the homogeneous equations of motion of the bicycle model lead to a characteristic equation which dictates that the motion of an oversteering vehicle is always a-periodic i.e. the roots of the characteristic equation are real and hence the time response is exponential [4]. Furthermore, the response of an oversteering vehicle becomes unstable when the forward velocity exceeds a critical value, as a result of one of the real roots becoming positive. On the other hand it is demonstrated that an under-steering vehicle is always stable, with a pair of real or complex roots determining the existence or not of oscillation before settling to a steady-state. This type of behaviour dominates the step-seer response of the linear bicycle model. At low forward speeds (below the critical speed), the response of the over-steering vehicle converges to steady-state in an exponential manner, whereas at velocities above the critical speed the motion diverges exponentially. The under-steering vehicle shows similar behaviour at low speeds, where the equivalent damping ratio approaches unity [2]. As the forward speed increases, the damping ratio drops [2] and the overshoot in the yaw response dominates the handling behaviour. In theory, this approach should answer all questions regarding the response of the vehicle to a step-steer manoeuvre. However, the assessment of the response in practice is not as straightforward. At modest forward speeds, the responses of neutral, under and over-steering vehicles are hard to distinguish. The overshoot of the yaw response of the under-steering vehicle deteriorates even for vehicles with a high under-steer coefficient [2] and it becomes difficult to classify a vehicle based on a single step-steer response. The situation is made 
worse when highly non-linear vehicle models are employed, or when the assessment is based on real vehicle data. In such cases the analysis of the vehicle's behaviour is more of a descriptive nature and the lack of robustly defined performance indices is evident [9],[10]. Finally, the explicit characterisation of the transient handling behaviour of a vehicle should ideally be possible when considering realistic steering inputs, as opposed to the mathematically simple step-steer manoeuvre. Such inputs would normally occur in full lap simulations or real race track data. It would then be desirable to be able to assess the sequence of the tendencies of a vehicle to under or over-steer during the course of a single manoeuvre.

The present work attempts to resolve a number of the aforementioned transient handling assessment issues. This aim is achieved with the development of a well structured methodology based on the combined use of two versions of a newly defined measure, namely the dynamic and kinematic normalised yaw impulses. In the following paragraphs the simple mathematical basis of the assessment is presented. It is demonstrated how the proposed methodology provides insight in the vehicle's behaviour at any time instant during the course of a transient manoeuvre. A number of case studies are carried out where the approach is used to analyse the influence of parameters which affect only the transient handling behaviour, and, finally, some limitations and general shortcomings of the method are commented upon.

\section{The dynamic normalised yaw impulse}

The concept of the normalised yaw impulse was first introduced in [11] for the rough assessment of the transient handling behaviour of a vehicle. Nevertheless, the qualities and the potential of this measure were not explored in depth. Considering a 
vehicle subjected to a transient manoeuvre, a definition of the general or dynamic yaw impulse is given below:

$\Gamma_{d}=\frac{|\mathrm{a}| \sum_{i=1,2} F_{y i} \cdot \mathrm{d} t-|\mathrm{b}| \sum_{i=3,4} F_{y i} \cdot \mathrm{d} t}{|\mathrm{a}| \sum_{i=1,2} F_{y i} \cdot \mathrm{d} t+|\mathrm{b}| \sum_{i=3,4} F_{y i} \cdot \mathrm{d} t}$

where the absolute values of the distances $a$ and $b$ will be used throughout the text to avoid confusion in the cases where $\mathrm{a}$ and $\mathrm{b}$ are stated as signed coordinates measured from the c.g.

The numerator in equation (1) represents the actual yaw impulse due to the tyre forces (with the positive yaw direction as defined by SAE [1]) while the denominator is equal to the vectorial addition of the front yaw impulse and the opposite vector to the rear yaw impulse. Apparently, the infinitesimally small amount of time $d t$ which justifies the use of the word 'impulse' can be omitted from equation (1) resulting in a ratio of yaw moments, not yaw impulses. It should be emphasized that each lateral force in equation (1) represents the projection of the net tyre force of a possibly steered and braking tyre on the $y$ axis of the SAE vehicle frame of reference.

By simply observing equation (1) some of the fundamental properties of the normalised yaw impulse become immediately apparent. For a front-steered vehicle, at the onset of any steering manoeuvre $\Gamma_{d}$ is equal to one, due to the second terms of both the numerator and denominator in equation (1) being initially equal to zero. On the other hand, if the steering is initiated at the rear, $\Gamma_{d}$ becomes equal to -1 . For a vehicle obtaining a steady-state motion after the application of a control input, $\Gamma_{d}$ converges to zero. This is easily explained if one considers the fact that under steady-state conditions 
the yaw acceleration and consequently the yaw moment (the numerator in equation (1)) converge to zero, while the denominator converges to a non-zero value.

The behaviour of the dynamic normalised yaw impulse is investigated further by implementing a linear 2 degree-of-freedom bicycle model. Assuming a constant forward speed $U$, sufficiently small steer angles and linear tyres, the equations of motion for the lateral and yaw degrees of freedom of the model read:

$$
\begin{aligned}
& \sum F_{y}=C_{f} \cdot a_{f}+C_{r} \cdot a_{r}=m(d V / \mathrm{d} t+U \cdot r) \\
& \sum M_{z}=|\mathrm{a}| C_{f} \cdot a_{f}-|\mathrm{b}| C_{r} \cdot a_{r}=I_{z z}(\mathrm{~d} r / \mathrm{d} t)
\end{aligned}
$$

Further assuming a front-steered vehicle and relatively small slip angles $a_{f}$ and $a_{r}$, the following relations apply [2]:

$$
\begin{aligned}
& a_{f}=\delta-\frac{V+|\mathrm{a}| r}{U} \\
& a_{r}=-\frac{V-|\mathrm{b}| r}{U}
\end{aligned}
$$

Equations (2) and (3) are solved numerically with the slip angles provided by equations (4) and (5). At each time-step, the dynamic normalised yaw impulse is calculated according to equation (1) and plotted against time. As a first case study, three vehicles with the same dimensions, inertial properties and front/rear mass distribution are subjected to a step-steer manoeuvre. The vehicles are made neutral, under and oversteering by adjusting the rear tyre stiffness accordingly. Table 1 contains all vehicle data and manoeuvre parameters. The words 'under', 'neutral' and 'over' are used to characterise the vehicles based on the value of the corresponding under-steer coefficient. Figures 1 and 2 show the yaw rate and lateral velocity responses of the three vehicles, while figure 3 shows the calculated normalised yaw impulse. The first 
observation is that the overshoot in the yaw response of the under-steer vehicle is eliminated due to the forward velocity being sufficiently low. Although a comparison of the three yaw rate curves reveals the ranking of the vehicles from the less to the most over-steering one, it is still hard to characterise each one of them in a definitive manner. The change in the sign of the lateral velocity of the over-steering vehicle might give some additional insight as to which one is the really over-steering one and, certainly, in this trivial case, simple system identification would probably reveal the actual properties of each one of the vehicles. Nevertheless, this is not the aim of this exercise, not to mention that such an approach is problematic with highly non-linear systems. The additional information provided by the normalised yaw impulse is not enlightening either. $\Gamma_{d}$ is initially equal to one, indicating front-steered vehicles, converges to zero in all three cases, indicating stable behaviour and consistently increases as the vehicle becomes more over-steering. This behaviour is related to the additional need for yaw moment (numerator in equation (1)), as the yaw rate of the over-steering vehicle increases further compared to that of the under or neutral-steering ones. However, the definitive characterisation of each vehicle is still not possible and the normalised yaw impulse can only be used as a comparative measure. Two more case studies reveal the influence of the moment of inertia on the transient handling performance and the reflexion of this influence in the normalised yaw impulse. Table 2 contains the data of three equally under-steering vehicles (based on the values of the under-seer coefficient) possessing different moments of inertia, while maintaining the same mass. Figures 4-6 show the corresponding responses and the normalised yaw impulse. The results indicate that, as the moment of inertia increases, the transient handling behaviour becomes more over-steering. This is primarily dictated by the yaw-rate response which appears to be more 'exponential' with a longer rise time [2]. The normalised yaw impulse also reflects 
the phenomenon, showing a consistent raise as the moment of inertia increases. In [2], certain simplifications have permitted the expression of the equivalent natural frequency and damping ratio independently of the moment of inertia. In reality, the influence of the moment of inertia requires further investigation. Starting from equation (2) and (3), elimination of the lateral velocity $V$ yields the following homogeneous equation for the yaw rate $r$ :

$m I_{z z} U^{2} \frac{d^{2} r}{\mathrm{~d} t^{2}}+m C U\left(q^{2}+k^{2}\right) \frac{d r}{\mathrm{~d} t}+C_{f} C_{r} l^{2}\left(1+\frac{K_{u s}}{g l} U^{2}\right) r=0$

where,

$C=C_{f}+C_{r}$

$C q^{2}=C_{f} \mathrm{a}^{2}+C_{r} \mathrm{~b}^{2}$

$m k^{2}=I_{z z}$

$K_{u s}=\frac{m|\mathrm{~b}| g}{l C_{f}}-\frac{m|\mathrm{a}| g}{l C_{r}}$

Since the yaw moment of inertia is altered without affecting the total mass, the changes are to be solely incorporated in the radius of inertia, $k$, which obtains the values 0.99 , 1.11 and $1.24 \mathrm{~m}$ respectively. Figure 7 shows how the roots of the corresponding characteristic equation vary as the radius of inertia shifts from $0.5 \mathrm{~m}$ to the rather unrealistic value of $2 \mathrm{~m}$. In the same graph the roots of the three discreet cases of table 2 are also shown. In addition, the values of the damping ratio and natural frequencies are shown in figure 8 . First, one needs to identify what constitutes a 'more over-steering' transient behaviour of an under-steering vehicle (based on its steady-state characterisation). Figure 7 indicates that, as the radius of inertia $k$ increases, the roots shift closer to the positive real axis, i.e. the yaw rate response becomes less stable. On 
the other hand, figure 8 indicates that the equivalent damping ratio reduces, a fact which points towards additional under-steer. The ambiguity of the situation is the result of the interrelation of the un-damped natural frequency and the damping ratio in the final expression of the yaw rate response. Referring back to figure 2, the yaw rate response points towards over-steer as already mentioned, with the normalised yaw impulse supporting the observation. The same tendency is observed in the case of three equally over-steering vehicles with different moments of inertia and equal masses (see table 3 and figures $9-11$ ). A possible conclusion is that the value of the real part of the roots is more representative of the transient cornering behaviour of a vehicle. Still, the approach is only comparative and the normalised yaw impulse does not provide significantly better insight than the yaw response alone. Following the previous analysis, the assessment of the transient handling behaviour will be aided further with the introduction of the kinematic yaw impulse.

\section{The kinematic normalised yaw impulse}

Starting from equation (1), the dynamic yaw impulse can be written in the following form for the linear bicycle model:

$$
\Gamma_{d}=\frac{|\mathrm{a}| C_{f} \delta-\frac{|\mathrm{a}| C_{f}}{U}(V+|\mathrm{a}| r)+\frac{|\mathrm{b}| C_{r}}{U}(V-|\mathrm{b}| r)}{|\mathrm{a}| C_{f} \delta-\frac{|\mathrm{a}| C_{f}}{U}(V+|\mathrm{a}| r)-\frac{|\mathrm{b}| C_{r}}{U}(V-|\mathrm{b}| r)}
$$

A parameter $\rho$ is introduced so that:

$$
\rho=\frac{|\mathrm{b}| C_{r}}{|\mathrm{a}| C_{f}}
$$


Note that, based on equations (10) and (12), the under-steer coefficient can be written as:

$$
K_{u s}=\frac{m|\mathrm{a}| g}{l C_{r}}(\rho-1)
$$

Clearly, for the under-steering vehicle $\rho>1$, for the neutral vehicle $\rho=1$ and, finally, the over-steering vehicle is characterised by $\rho<1$.

With the introduction of parameter $\rho$, equation (11) can be simplified as follows:

$$
\Gamma_{d}=\Gamma_{k}=\frac{U \delta-V(1-\rho)-r(|\mathrm{a}|+\rho|\mathrm{b}|)}{U \delta-V(1+\rho)-r(|\mathrm{a}|-\rho|\mathrm{b}|)}
$$

Equation (14) represents the kinematic form of the normalised yaw impulse, denoted $\Gamma_{k}$ in the remaining text. Note that for the neutral bicycle model, $\Gamma_{k}$ can be simplified further:

$$
\Gamma_{k n}=\frac{U \delta-r l}{U \delta-2 V-r(|\mathrm{a}|-|\mathrm{b}|)}
$$

Relation (15) will form the basis for the characterisation of the handling behaviour, as discussed in detail in the following section.

\section{$4 \quad$ Assessment of the transient handling behaviour}

Thus far, both the dynamic and kinematic expressions of the normalised yaw impulse have been presented. The assessment of the handling behaviour will be achieved through a structured methodology, based on the combined use of these measures and four fundamental axioms.

Axiom 1: 
'The linear two-degree-of-freedom bicycle model with immediate tyre response (zero phase lags in the build-up of tyre forces) which possesses an under-steer coefficient equal to zero, is declared to behave neutrally in terms of handling, under all possible operating conditions, steady-state or transient.'

Axiom 2:

'If a vehicle, linear or non-linear, at any instant during the course of a transient manoeuvre, exhibits a dynamic yaw impulse $\Gamma_{d}$ equal to $\Gamma_{k n}$, the vehicle at this particular instant steers neutrally.'

\section{Axiom 3:}

'If a vehicle, linear or non-linear, at any instant during the course of a transient manoeuvre, exhibits a dynamic yaw impulse $\Gamma_{d}$ larger than $\Gamma_{k n}$, the vehicle at this particular instant over-steers.'

Axiom 4:

'If a vehicle, linear or non-linear, at any instant during the course of a transient manoeuvre, exhibits a dynamic yaw impulse $\Gamma_{d}$ smaller than $\Gamma_{k n}$, the vehicle at this particular instant under-steers.' 
The methodology for resolving the problem of the assessment of the transient handling behaviour has now become apparent. Considering a vehicle subjected to a transient handling manoeuvre, the velocity traces together with the steer-angle history can be used in equation (15) to calculate the kinematic normalised yaw impulse of an assumedly neutral vehicle. The validity of the assumption of a neutral vehicle is then checked by comparing the value of $\Gamma_{k n}$ with the value of the dynamic normalised yaw impulse $\Gamma_{d}$ at every time instant during the course of the manoeuvre. Mathematically, the comparison can be achieved in a number of ways. Probably the simplest approach is by calculating the following ratio:

$Q=\frac{\Gamma_{d}}{\Gamma_{k n}}$

In the event that $Q=1$ the vehicle behaves neutrally. If $Q<1$ it exhibits under-steer, whereas if $Q>1$ the vehicle over-steers.

Although equation (16) provides a generally satisfactory means for characterising the handling behaviour, some problems exist, with certain types of manoeuvres. In particular, it will be shown through simulation, that, under certain circumstances, $\Gamma_{d}$ and/or $\Gamma_{k n}$ become negative. The $Q$ ratio then fails to compare the two measures and its usefulness deteriorates. On the other hand, the direct calculation of the difference between $\Gamma_{d}$ and $\Gamma_{k n}$ would be insensitive to sign changes, nevertheless it fails to show the relative magnitude of $\Gamma_{d}$ against $\Gamma_{k n}$. In addition, while experimenting with a variety of expressions containing combinations of ratios, differences and absolute 
values, it was found that numerical problems exist when $\Gamma_{d}$ and/or $\Gamma_{k n}$ approach zero. To tackle such situations, a second measure is employed, as described by the relation below:

$$
Q_{s}=\frac{\left(\Gamma_{d}-\Gamma_{k n}\right) B_{1}}{\left|\Gamma_{d}-\Gamma_{k n}\right|+B_{2}}
$$

In equation (17), $B_{1}$ and $B_{2}$ are Boolean expressions yielding 1 (true) or 0 (false) according to the following relations:

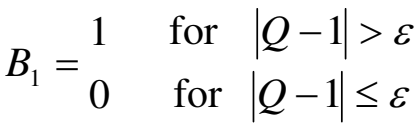

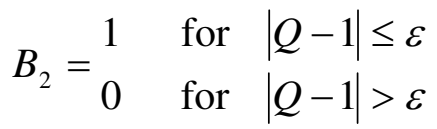

where $\varepsilon$ is the 'neutral margin', dictating the maximum relative difference allowed between $\Gamma_{d}$ and $\Gamma_{k n}$, before classifying a transient response as non-neutral.

In the event that $|Q-1|=\left|\left(\Gamma_{d}-\Gamma_{k n}\right) / \Gamma_{k n}\right|>\varepsilon$, the relative difference between $\Gamma_{d}$ and $\Gamma_{k n}$ is such that the vehicle cannot be characterised as neutral. Under such conditions $B_{1}=1, B_{2}=0$ and $Q_{s}$ represents the sign of the difference between $\Gamma_{d}$ and $\Gamma_{k n}$. If $Q_{s}=-1$ the vehicle exhibits under-steer, whereas if $Q_{s}=1$ it over-steers. Alternatively, 
if $|Q-1|=\left|\left(\Gamma_{d}-\Gamma_{k n}\right) / \Gamma_{k n}\right| \leq \varepsilon$ the vehicle is considered neutral, $B_{1}=0, B_{2}=1$ and $Q_{s}=0$

Equations (17-19) provide a robust criterion for the explicit characterisation of a vehicle's handling response under any possible transient input. The main disadvantage of $Q_{s}$ is the 'one and a half bit' of information it offers. $Q_{s}$ can only tell whether a vehicle is neutral, over or under-steering, but fails to determine by what amount. Still, numerous simulations have shown that this information can be effectively combined with the actual responses of the vehicle in order to gain better insight. Especially with non-linear vehicles, the point in the time history when the response switches from neutral to under/over steering, provides additional information about the handling characteristics of the vehicle. Moreover, having identified the weakness of the $Q$ ratio as a handling index, it can prove helpful when used with judgement for the quantification of the amount of over or under-steer under certain transient manoeuvres.

The assessment of the transient handling behaviour is demonstrated in practice by applying this methodology to the three test cases discussed in the previous section. In all cases the neutral margin $\varepsilon$ for the calculation of $Q_{s}$ is set equal to 0.01 .

Figure 12 shows the neutral kinematic yaw impulse $\Gamma_{k n}$ as calculated using the velocities and steering angle information for the three hypothetical vehicles of table 1 . Although all three responses to the step-steer input are stable (see figures 1,2), $\Gamma_{k n}$ converges to zero only in the neutral case. This is due to the fact that $\Gamma_{k n}$ equals the actual dynamic yaw impulse depicted in figure 3 only for the neutral vehicle. On the 
contrary, in the under-steering case $\Gamma_{k n}$ reduces less rapidly, converging to a value greater than zero. Finally, in the over-steering case $\Gamma_{k n}$ reduces faster and converges to a negative steady-state value. The usefulness and the limitations of the $Q$ ratio are clearly demonstrated in figure 13 and the corresponding detail depicted in figure 14 . The $Q$ ratio is effectively calculated by dividing the $\Gamma_{d}$ curves as shown in figure 3 with the $\Gamma_{k n}$ curves in figure 12. The double-spike pattern characterising the oversteering vehicle in figure 13 is the result of $\Gamma_{k n}$ approaching zero with a relatively large negative slope and subsequently becoming negative, as shown in figure 12. Obviously, the area around the spikes has no physical significance and the magnified detail in figure 14 indicates that the $Q$ ratio can probably be used as an index up to a maximum of 0.2 seconds after the initiation of the manoeuvre. Also, it is demonstrated that the $Q$ ratio remains consistently equal to one for the neutral vehicle. The 'one and a half bit' of information that the $Q_{s}$ ratio offers is depicted in figure 15 . For a very short period at the beginning of the manoeuvre, all vehicles exhibit a neutral behaviour, as a result of the quantity $|Q-1|$ being smaller than the chosen neutral margin $\varepsilon$. For the under and over-steering vehicles the neutral margin $\varepsilon$ is quickly exceeded and the $Q_{s}$ ratio equals 1 and -1 , respectively.

The $Q$ and $Q_{s}$ ratios for the three equally under-steering vehicles (based on the understeer coefficient) with different radii of inertia (see table 2) are shown in figures 16 and 17, respectively. The curves in figure 16 reveal the usefulness of the $Q$ ratio in quantifying the transient handling behaviour. It is shown that as the radius of inertia and subsequently the yaw moment of inertia increases, the $Q$ ratio increases as well, 
approaching that of the corresponding neutral vehicle. This observation can be interpreted as follows:

'The response of an under-steering vehicle to a step-steer manoeuvre moves closer to the response of the corresponding neutral vehicle, as the radius of yaw moment of inertia increases'.

Attention should be paid to the underpinning implications of the above interpretation. The assessment of the handling behaviour is based on the calculation of $\Gamma_{k n}$ of an assumedly neutral vehicle using the velocity traces and steering angle of the actual vehicle. Hence, it must be emphasised, that, the reference to a 'corresponding neutral vehicle' points to the instantaneous, assumedly neutral response of the vehicle, not to an assumedly neutral vehicle subjected to the same manoeuvre in parallel to the actual vehicle. An alternative approach is provided to further clarify the situation: $\Gamma_{k n}$ can be perceived as a purely kinematic measure which is calculated for any vehicle at any point of time, under any transient manoeuvre. Only if the response is a neutral one, will the kinematic measure $\Gamma_{k n}$ coincide with the dynamic measure $\Gamma_{d}$. The greater the relative difference between $\Gamma_{k n}$ and $\Gamma_{d}$ (expressed by ratio $Q$ ), the further the gap between the observed response and the neutral one. Finally, the $Q_{s}$ ratios depicted in figure 17, provide a definite characterisation of the three handling responses, rating all three of them as under-steering. At this point, the $Q$ ratio provides a more integrated picture of the handling responses of the hypothetical vehicles, compared to that of the $Q_{s}$ ratio. Not only does it classify all vehicles as under-steering $(Q<1)$, it also enables the 
comparison between them and can be used to rank their behaviour starting from the least to the most under-steering one.

The case of three equally over-steering vehicles (see Table 3) is dealt with in figures 18 and 19. This time the usefulness of the $Q$ ratio deteriorates quickly and only a small part of the response, say up to $0.15 \mathrm{~s}$ after the initiation of the manoeuvre, can be employed for comparing and ultimately ranking the vehicles. Otherwise, it is demonstrated that the $Q_{s}$ ratio provides a definitive characterisation throughout the course of the manoeuvre. In terms of the interpretation of the results, as the radius of the yaw moment of inertia increases, the vehicle response moves closer to that of the 'corresponding' or 'assumedly' neutral vehicle, just like in the previous, under-steering case.

The overall conclusion that might be drawn is straightforward: Having de-coupled the yaw moment of inertia from the mass of the vehicle in order to avoid altering the understeer coefficient (equation 10), the increase in the yaw moment of inertia leads, in general, to a more neutral behaviour. This result seems to be in conflict with the implications of figures 4 and 7-10 and the analysis in section 2. However, in reality the results are not directly comparable and the conflict may be characterised as ostensible. In particular, figures 4 and 7-10 compare three vehicles with different yaw moments of inertia (different radii of moment) with each-other, while figures 16 and 18 compare each one of the vehicles with an assumedly neutral vehicle. The $\Gamma_{k n}$ responses of the assumedly neutral vehicles are unique in each case and, as discussed previously, incorporate the influence of the vehicle parameters in an indirect manner, i.e. by using in the calculations the velocity responses of the actual vehicle. Undoubtedly, the 
question to be answered, is, whether it is useful to classify a response as 'closer to neutral' (see for example the dashed curve in figure 16), when at the same time figure 4 indicates that the response of this specific vehicle is the most over-steering one, possessing the longest rise time. The answer to this question initiates from the observation that even a purely neutral vehicle - based on steady-state criteria - would respond in a more over-steering manner, as its radius of yaw moment of inertia increases. This is shown in figure 20 which depicts the yaw-rate response of the neutral vehicle from Table 1, with its yaw moment of inertia changing between three different values. The previous question can now be rephrased as to whether it is sensible to characterise a response of a neutral vehicle as more or less under/over-steering, compared to the response of another neutral vehicle. Such an approach would adversely affect the definition of an absolute benchmark for the assessment of the handling behaviour, as attempted with the $1^{\text {st }}$ axiom in section 4 . Secondly, although the steadystate case can be dealt with separately from any transient manoeuvre, the relativity introduced in the classification of the transient behaviour of neutral vehicles would create an unwanted conflict with the fact that all neutral vehicles behave equally neutrally under steady-state conditions. To conclude, the underlying aim of the proposed handling assessment methodology is the de-coupling of the assessment from the primary motion responses of the vehicle. By employing the dynamic and kinematic measures $\Gamma_{d}$ and $\Gamma_{k n}$, the effect of the moment balance between the front and the rear parts of the vehicle is built into the assessment process, much like the difference between front and rear slip angles is built into the assessment of steady-state cornering. Following this analysis, it can be observed that the increase in the yaw moment of inertia of a neutral vehicle does not alter the balance of moments about its z-axis, therefore the $Q$ ratio always equals 1 and the vehicle is always classified as neutral. On 
the contrary, in the case of both under and over-steering vehicles, different yaw moments of inertia alter the balance of moments about the z-axis during the course of the manoeuvre. This results in the vehicles becoming more or less under/over-steering, as dictated by the $Q$ ratio. A possible steady-state equivalent is the influence of a vehicle's mass on its cornering behaviour. In particular, altering the mass of a neutral vehicle does not alter the difference between front and rear slip angles, while negotiating a corner of given radius. In contrast, changes in the mass of the vehicle affect the under-steer coefficient of both under and over-steering vehicles and consequently the difference between front and rear slip angles, while negotiating a corner of given radius. Finally, to further support the predictions of figs. 16 and 18 and prove their physical significance, three neutral vehicles with increasing radius of yaw moment were run in parallel to the three under and over-steering ones, subjected to an identical step-steer manoeuvre. This, off course, does not imply that the responses of the three neutral vehicles are equivalent to the responses of the assumedly neutral vehicles employed in the calculation of the $Q$ ratio in figs. 16 and 18 . The latter are completely theoretical entities and signify the assumedly neutral instantaneous response of the actual vehicles. However, in the simple case of a step steer manoeuvre performed with linear vehicles, the difference, for instance, between the yaw response of the neutral vehicle with small inertia and the equivalent under/over-steering vehicle, should be greater than the difference between the corresponding vehicles with high inertia. To demonstrate this, the error/difference between the neutral and the under/over-steering yaw-rate responses throughout the first 0.8 seconds of the manoeuvre are calculated using equation (20) and included in Table 4. 
error $=\sqrt{\frac{\int_{0}^{\Delta t}\left(r_{\text {neutral }}-r_{\text {under/over }}\right)^{2}}{\Delta t}}$, with $\Delta t=0.8 \mathrm{~s}$

Hitherto, the step-steer manoeuvre has dominated the analysis. Similar results have been found in the case of a ramp-steer manoeuvre, however, as a final test case, the somewhat more interesting response to a sinusoidal steering input is briefly presented. Figure 21 shows the resulting yaw rate response of the three hypothetical vehicles of Table 1, subjected to a sinusoidal steering with amplitude equal to that of the step-steer manoeuvre $(0.02 \mathrm{rad})$ and a frequency of $0.5 \mathrm{~Hz}$. Figures 22 and 23 show the $Q$ and $Q_{s}$ ratios, respectively. The deterioration of the $Q$ ratio as a handling index under such operating conditions is immediately evident by observing figure 22 . On the other hand, the robust $Q_{s}$ ratio reveals the fact that only the neutral vehicle remains neutral throughout the course of the manoeuvre, while the responses of both the under and over-steering vehicles change suddenly from under to over-steering and vice-versa. This odd prediction is easily explained considering, for the sake of argument, the response of the under-steering vehicle. According to the $Q_{s}$ ratio, the vehicle is predicted to understeer up to approximately half a period of the excitation. During that time, the steerangle is positive. Because the vehicle under-steers, towards the end of the half period it possesses a less 'steered' state than the state it would possess if it were neutral. As the steering starts to become negative, the less 'steered' vehicle is ready to steer to the opposite direction more than it would if it were neutral, i.e. the vehicle temporarily over-steers. After a short period, equilibrium is re-established and the vehicle exhibits under-steering behaviour for the rest of the negative steering, until, approximately, the end of a full steering cycle. A similar analysis applies to the over-steering vehicle. An 
important observation is that the sudden changes in the responses of both non-neutral vehicles cover a small fragment of the full steering cycle. Otherwise, most of the time during the cycle, the characterisation of the transient response of the vehicles agrees with their steady-state classification as over or under-steering. This example reveals the importance of an additional parameter, that is, the amount of time required before the $Q_{s}$ ratio switches between different values during the course of a manoeuvre.

\section{$5 \quad$ Conclusions}

A structured methodology has been presented for the objective assessment of the transient handling response of a vehicle. Especially in the cases where an explicit, quantitative assessment is required, a benchmark needs to be defined axiomatically. The effectiveness of the choice of benchmark and the choice of methods used to compare the behaviour of the specimens to the behaviour of the benchmark is ultimately judged by the consistency, usefulness and practical application of the comparison results. In the present work, the benchmark and the comparison approaches are defined by the four axioms in section 4 and extended in the subsequent analysis. A number of simulations have been employed for the demonstration of the application of the assessment process in practice. Various implications have been explained in depth and the results of the method appear to be consistent and meaningful. The linear bicycle model was employed for the sake of simplicity and to enhance understanding of the basic principles of the method, however the neutral vehicle as defined by axiom 1 and the related kinematic measure $\Gamma_{k n}$ are to be used for the assessment of the behaviour of non-linear vehicles alike. Such work has already been undertaken and will form the basis of a second publication, hopefully to be released in due course. 
While the proposed method is readily applicable in simulation studies, there are certain practical difficulties related to the experimental measurement of the instantaneous tyre forces required for the calculation of $\Gamma_{d}$. Fortunately, instrumented wheel hubs are available in the market and have been used successfully [12] for the in-situ measurement of tyre forces. Furthermore, the solution to this practical challenge might prove easier than initially anticipated, if one realises that not all four lateral tyre forces are required, since, the combined front and rear axle forces suffice for the calculation of $\Gamma_{d}$. These in turn can be calculated indirectly using the kinematic responses (accelerations) of the vehicle and the corresponding inertias. In any case, further studies are required to reveal the strengths and weaknesses of the proposed methodology and to explore all possible applications.

\section{List of symbols}

a

b

$B_{1}, B_{2}$

$C_{f}$

$C_{r}$

$F_{y i}$

$g$

$I_{z z}$

$k$

$K_{u s}$

$l$
Distance of the c.g. from the front axle

Distance of the c.g. from the rear axle

Boolean expressions

Cornering stiffness of front tyres

Cornering stiffness of rear tyres

Lateral force at the $\mathrm{i}^{\text {th }}$ corner of a vehicle

Acceleration of gravity

Yaw moment of inertia

Radius of yaw moment of inertia

Under-steer coefficient

Wheelbase 
$m$

$Q, Q_{s}$

$r$

$U$

V

\section{Greek symbols}

$a_{f}$

$a_{r}$

$\Gamma_{d}$

$\Gamma_{k}$

$\Gamma_{k n}$

$\delta$

$\varepsilon$

$\rho$

\section{Subscripts}

neutral

under / over
Mass of the vehicle

Ratios

Yaw rate

Forward velocity

Lateral velocity
Front slip angle

Rear slip angle

Dynamic normalised yaw impulse

Kinematic normalised yaw impulse

Neutral kinematic norm. yaw impulse

Steer angle

Neutral margin

parameter
Indicates neutral vehicle

Indicates under/over-steering vehicle

\section{References}

[1] Vehicle Dynamic Terminology, SAE J670e, 1976.

[2] Pacejka H. B. Tyre and Vehicle Dynamics, 2002 (Butterworth Heinemann), Oxford. 
[3] Pacejka, H. Simplified Analysis of Steady-State Turning Behaviour of Motor Vehicles. Part 1: Handling diagrams of Simple Systems. Vehicle System Dynamics, 1973, 2, pp. 161-172.

[4] Pacejka, H. Simplified Analysis of Steady-State Turning Behaviour of Motor Vehicles. Part 2: Stability of the Steady State Turn. Vehicle System Dynamics, 1973, 2, pp. 173-183.

[5] Pacejka, H. Simplified Analysis of Steady-State Turning Behaviour of Motor Vehicles. Part 3: More Elaborate Systems. Vehicle System Dynamics, 1973, 2, pp. 185204.

[6] Lukowski S.A., Fiedler R.A. and Claar P.W. An Investigation of a Road-Vehicle Directional Behaviour Under Steady-State Conditions. SAE paper 911872, 1991.

[7] Allen, R.W., Rosenthal, T.J. and Szostak, H.T. Steady State and Transient Analysis of Ground Vehicle Handling. SAE paper 870495, 1987.

[8] Dutton, K., Thomson, S. and Barraclough, B. The art of control engineering, 1997 (Addison Wesley Longman), Essex.

[9] Lee, Yung-Li, Dziuba, J.C. Jr. and Lu, Ming-Wei. Vehicle handling design process using DOE. International Journal of Vehicle Design, 1996, 17(1), pp. 40-54.

[10] Antoun, R.J., Hackert, P.B., O’Leary, M.C. and Sitchin, A. Vehicle dynamic handling computer simulation: Model development, correlation and application using ADAMS. SAE paper 860574, 1986. 
[11] Mavros, G., Tyre Models for Vehicle Handling Analysis under Steady-State and Transient Manoeuvres, 2005, PhD Thesis, Loughborough University.

[12] Canudas-De-Wit C., Tsiotras P., Velenis E., Basset M. and Gissenger G. Dynamic Friction Models for Road/Tire Longitudinal Interaction. Vehicle System Dynamics, 2003, 39, pp. 189-226. 
Figure captions:

Figure 1: Yaw rate response to a step-steer manoeuvre of an under, neutral and oversteering vehicle

Figure 2: Lateral velocity response to a step-steer manoeuvre of an under, neutral and over-steering vehicle

Figure 3: Dynamic normalised yaw impulse response to a step-steer manoeuvre of an under, neutral and over-steering vehicle

Figure 4: Yaw rate response to a step-steer manoeuvre of three equally under-steering vehicles with different radii of yaw moment of inertia

Figure 5: Lateral velocity response to a step-steer manoeuvre of three equally understeering vehicles with different radii of yaw moment of inertia

Figure 6: Dynamic Normalised yaw impulse response to a step-steer manoeuvre of three equally under-steering vehicles with different radii of yaw moment of inertia

Figure 7: Root locus for three equally under-steering vehicles with different radii of yaw moment of inertia

Figure 8: Equivalent damping ratio and natural freqs. for three equally under-steering vehicles with different radii of yaw moment of inertia

Figure 9: Yaw rate response to a step-steer manoeuvre of three equally over-steering vehicles with different radii of yaw moment of inertia

Figure 10: Root locus for three equally over-steering vehicles with different radii of yaw moment of inertia

Figure 11: Equivalent damping ratio and natural freqs. for three equally over-steering vehicles with different radii of yaw moment of inertia 
Figure 12: Neutral kinematic normalised yaw impulse response to a step-steer manoeuvre of an under, neutral and over-steering vehicle

Figure 13: $Q$ ratio response to a step-steer manoeuvre of an under, neutral and oversteering vehicle

Figure 14: Detail from figure 13

Figure 15: $Q_{s}$ ratio response to a step-steer manoeuvre of an under, neutral and oversteering vehicle

Figure 16: $Q$ ratio response to a step-steer manoeuvre of three equally under-steering vehicles with different radii of yaw moment of inertia

Figure 17: $Q_{s}$ ratio response to a step-steer manoeuvre of three equally under-steering vehicles with different radii of yaw moment of inertia

Figure 18: $Q$ ratio response to a step-steer manoeuvre of three equally over-steering vehicles with different radii of yaw moment of inertia

Figure 19: $Q_{s}$ ratio response to a step-steer manoeuvre of three equally over-steering vehicles with different radii of yaw moment of inertia

Figure 20: Yaw rate response to a step-steer manoeuvre of three equally neutral vehicles with different radii of yaw moment of inertia

Figure 21: Yaw rate response to a sinusoidal steering input of an under, neutral and over-steering vehicle

Figure 22: $Q$ ratio response to a sinusoidal steering input of an under, neutral and oversteering vehicle

Figure 23: $Q_{s}$ ratio response to a sinusoidal steering input of an under, neutral and oversteering vehicle 
Table captions:

Table 1: Simulation data (case study 1)

Table 2: Simulation data (case study 2)

Table 3: Simulation data (case study 3)

Table 4: Influence of the radius of yaw moment of inertia on the steering behaviour 


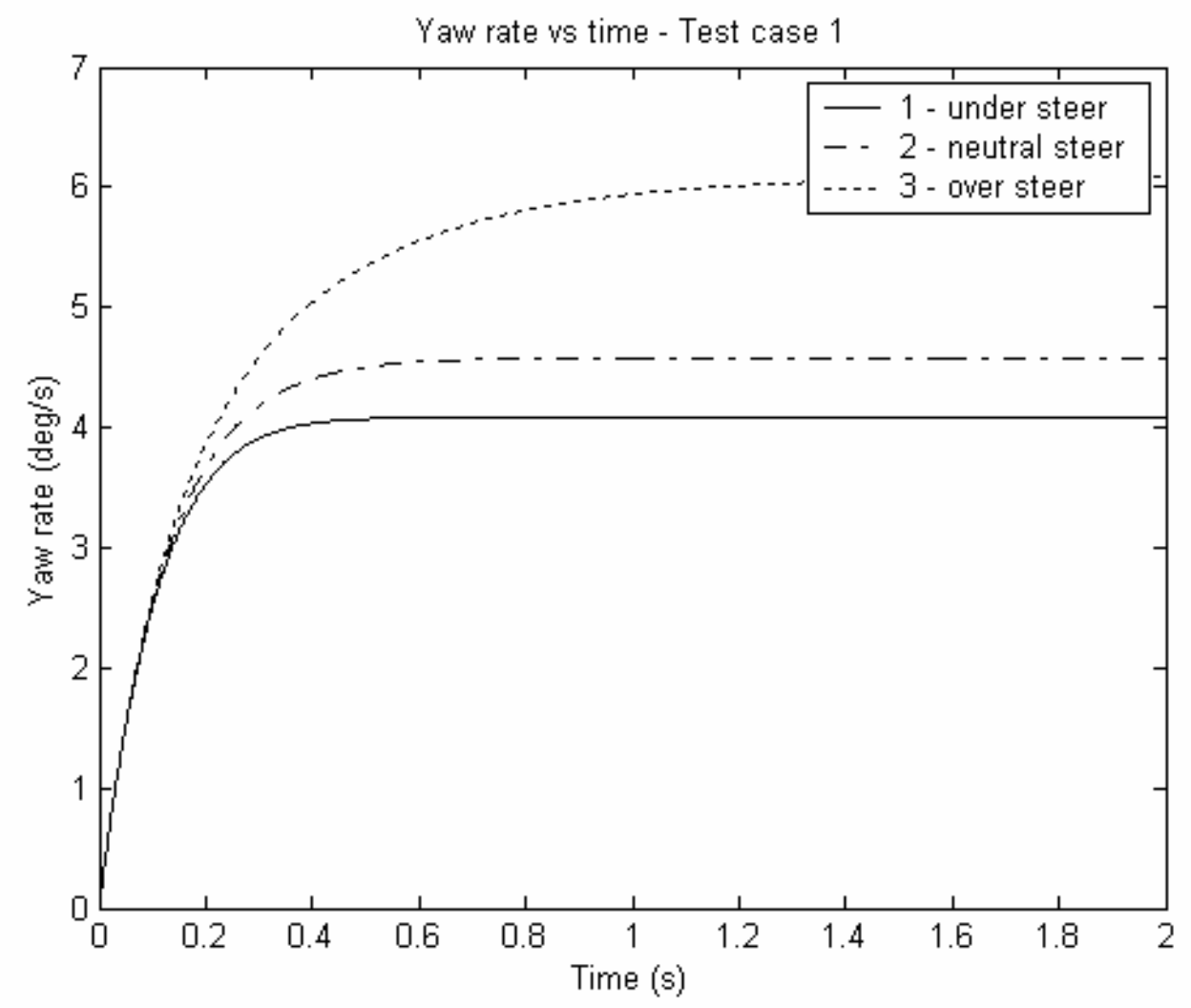

Figure 1 


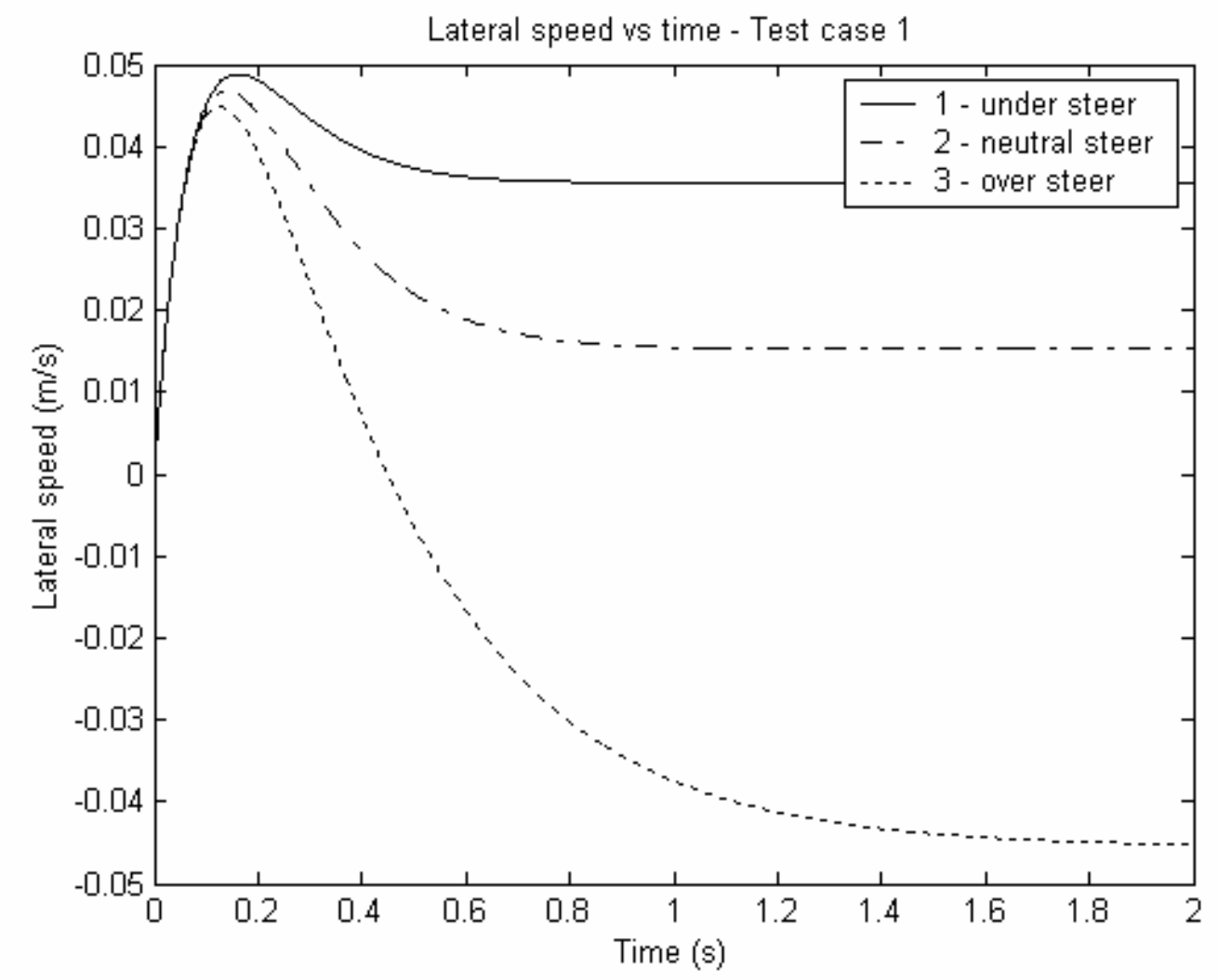

Figure 2 


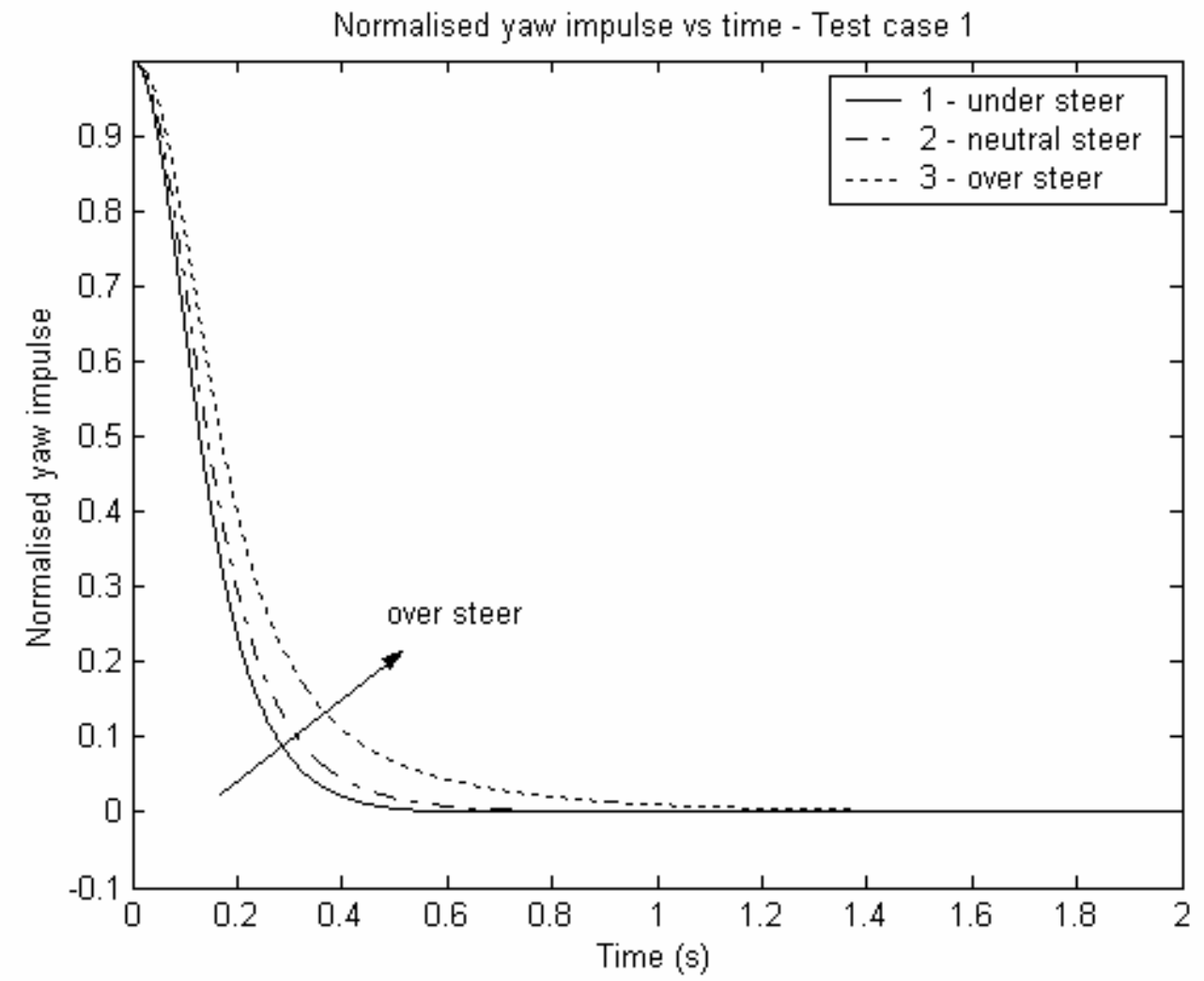

Figure 3 


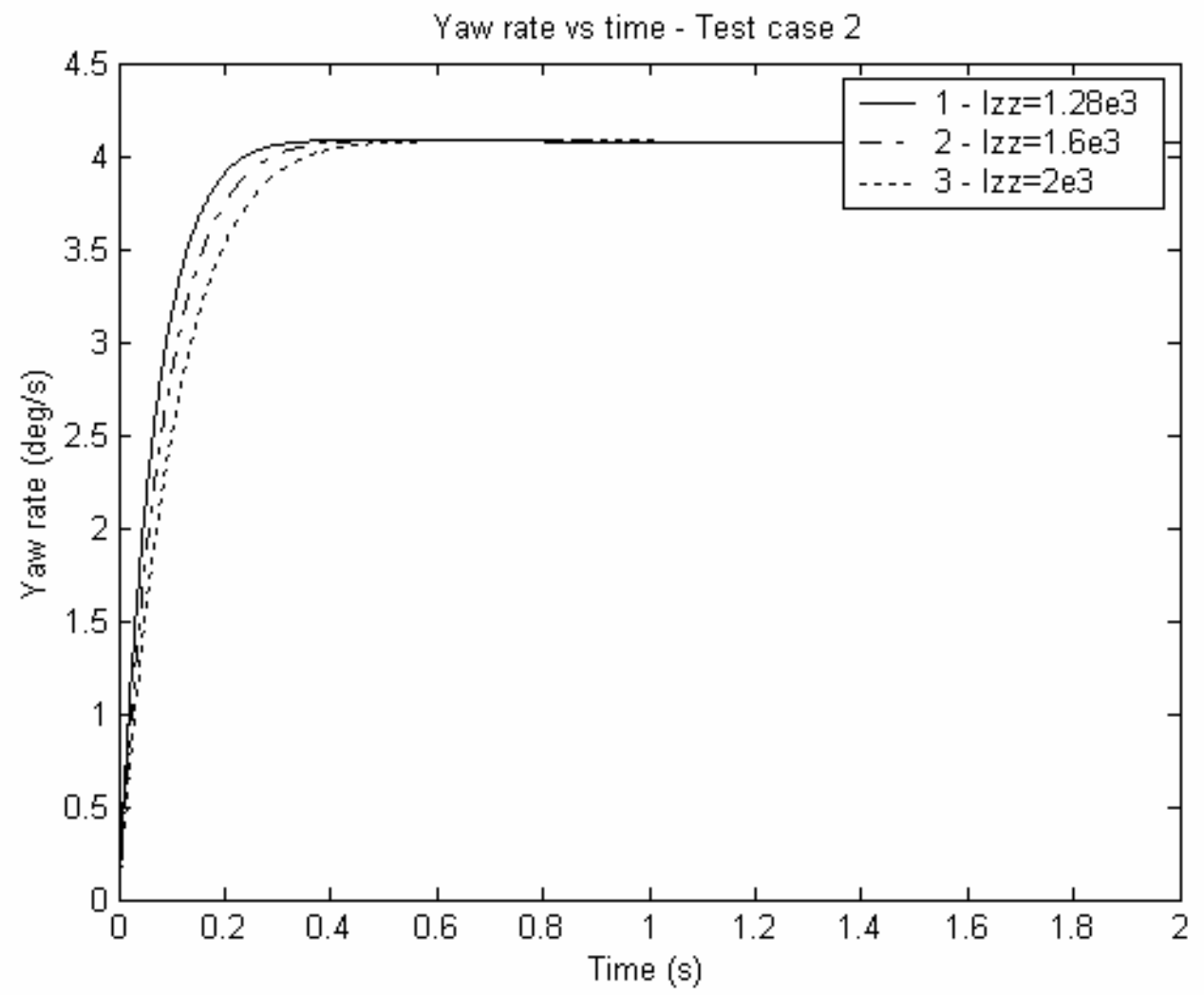

Figure 4 


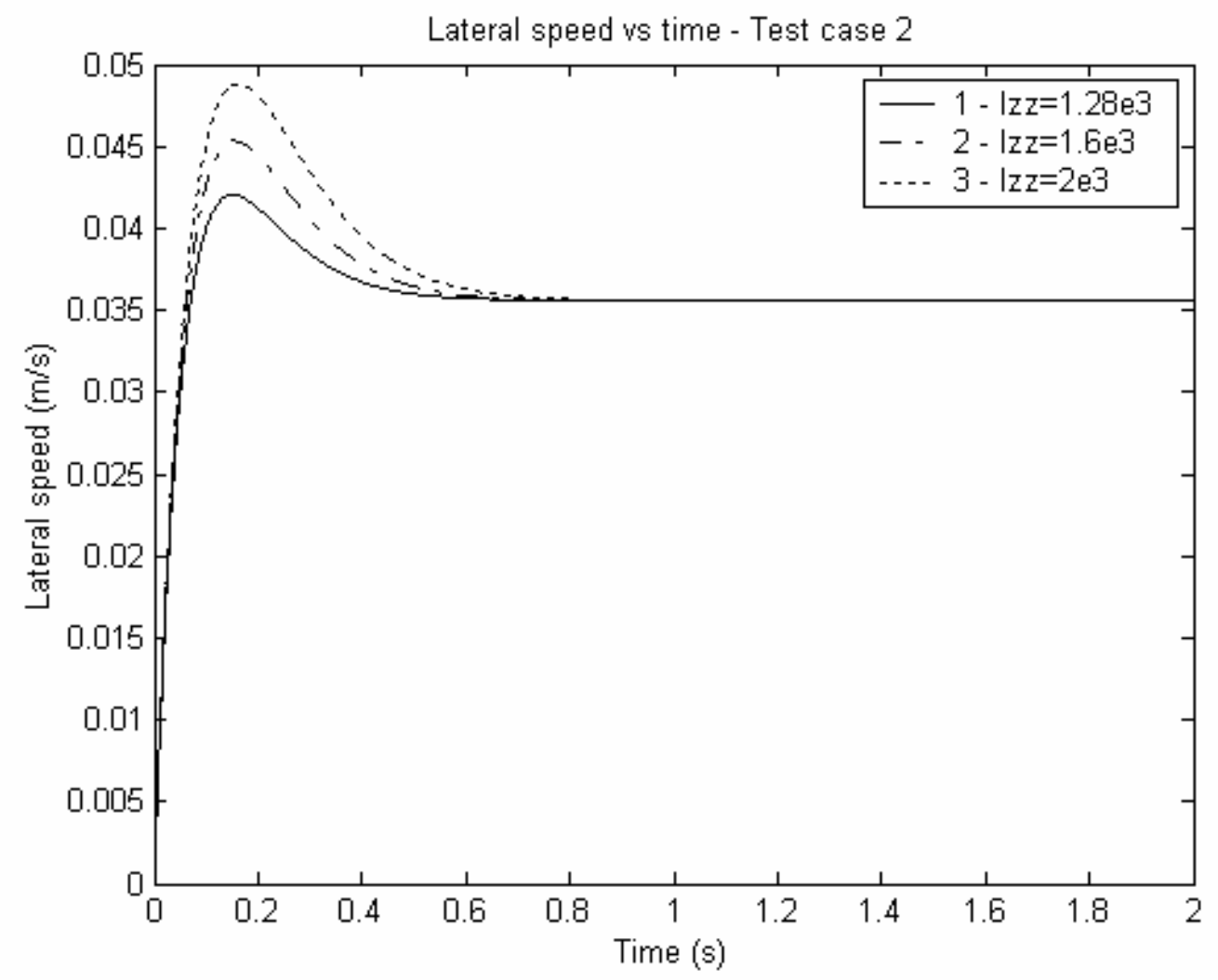

Figure 5 


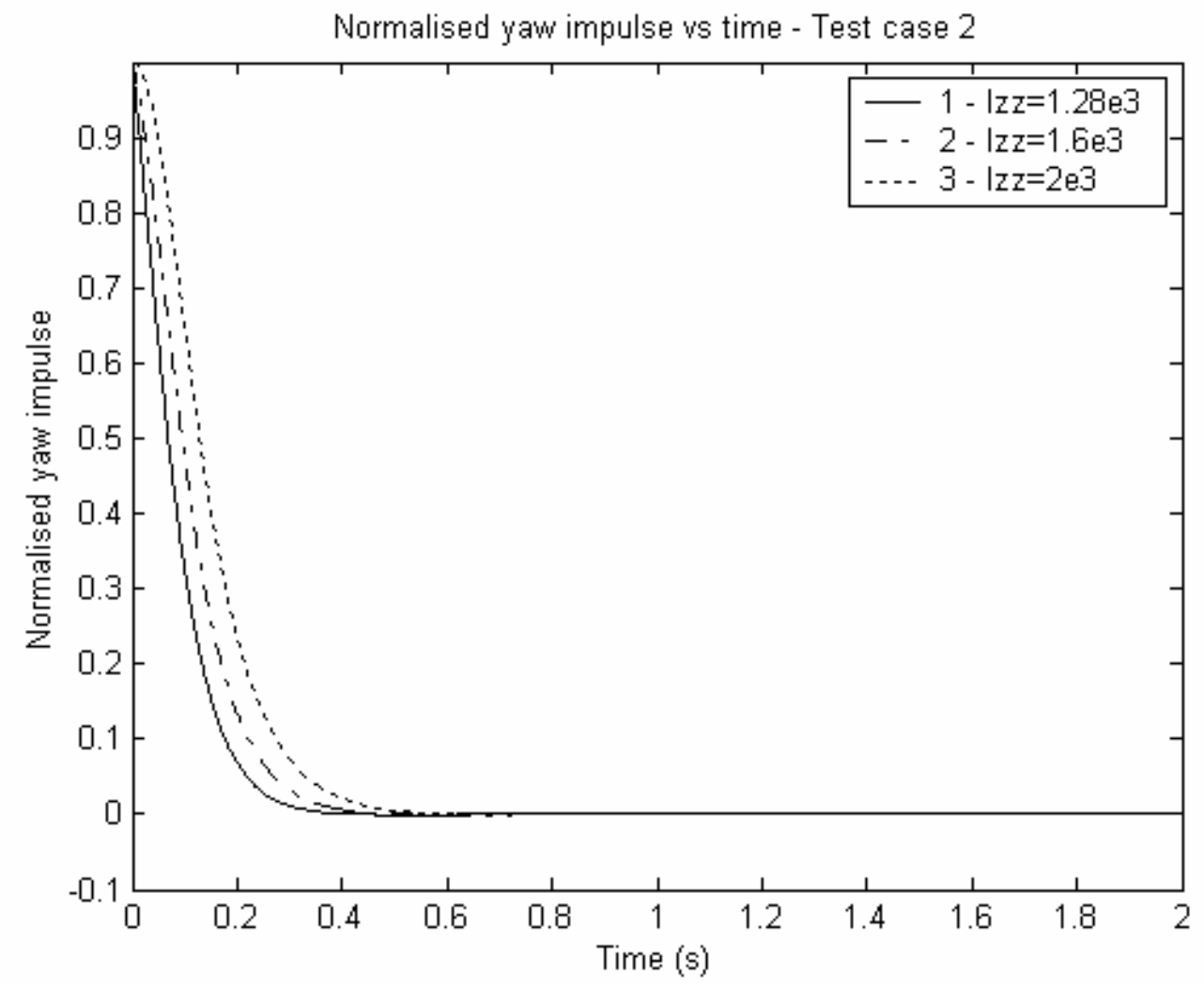

Figure 6 
Roots of the characteristic equation of the yaw response - Test case 2

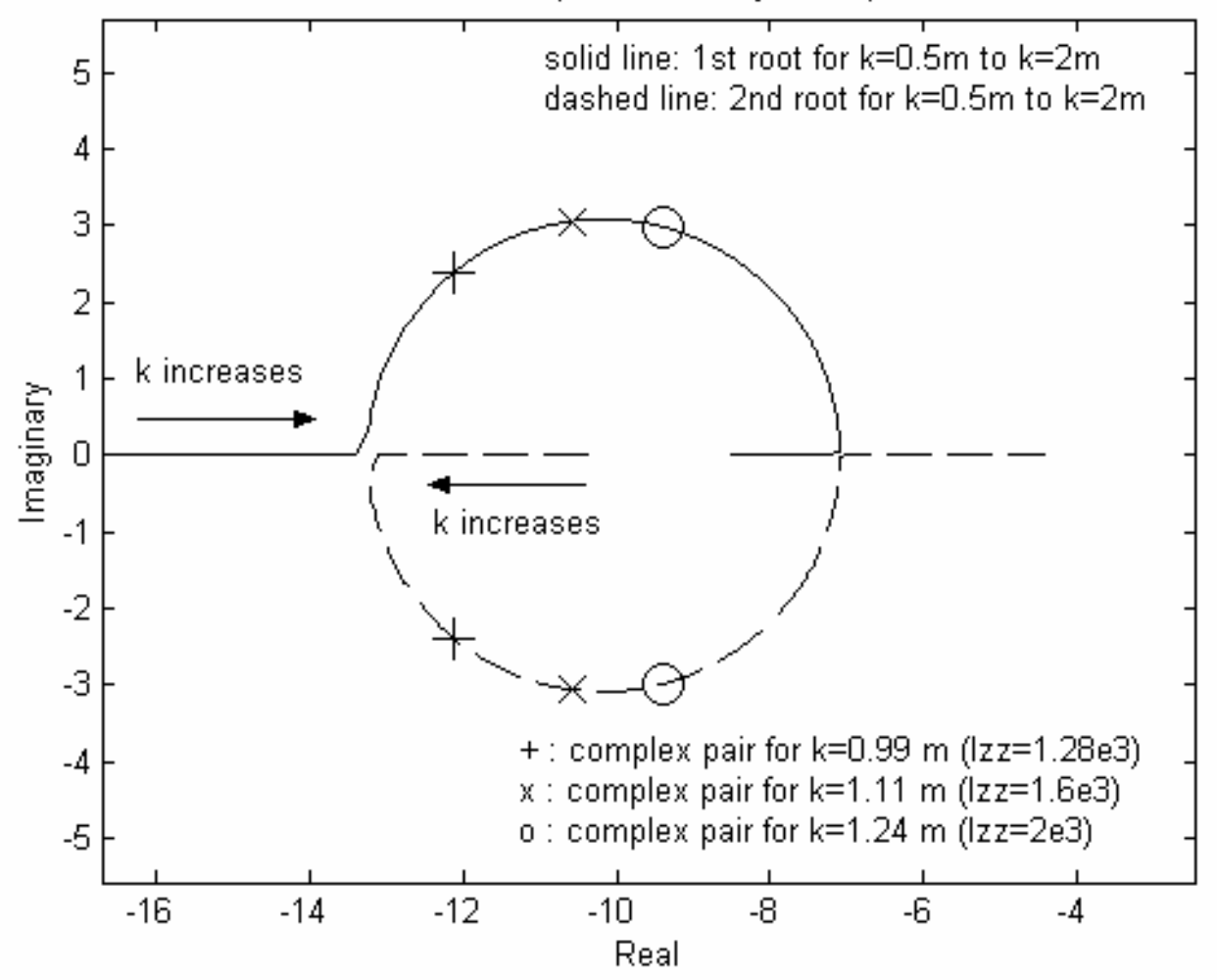

Figure 7 


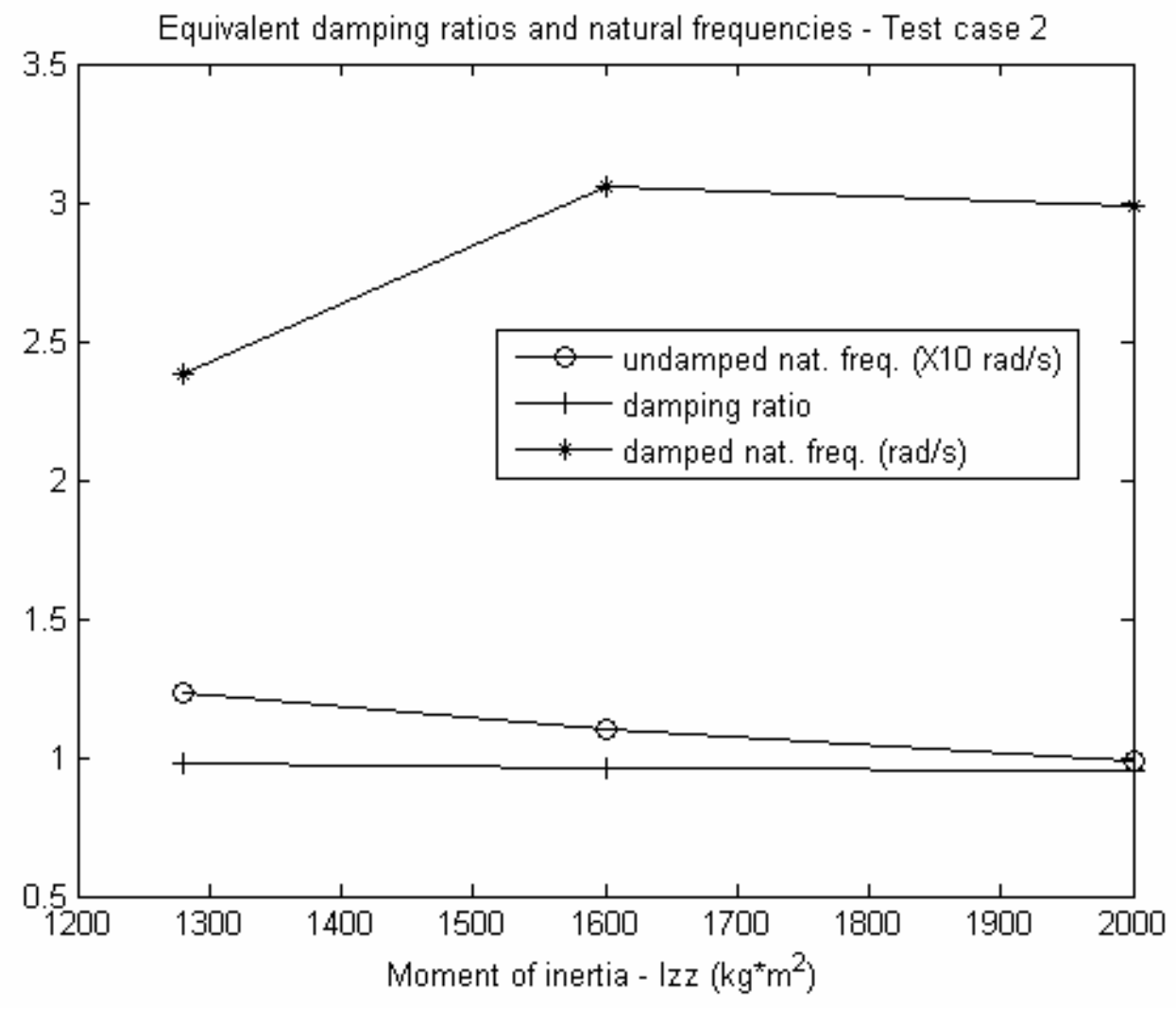

Figure 8 


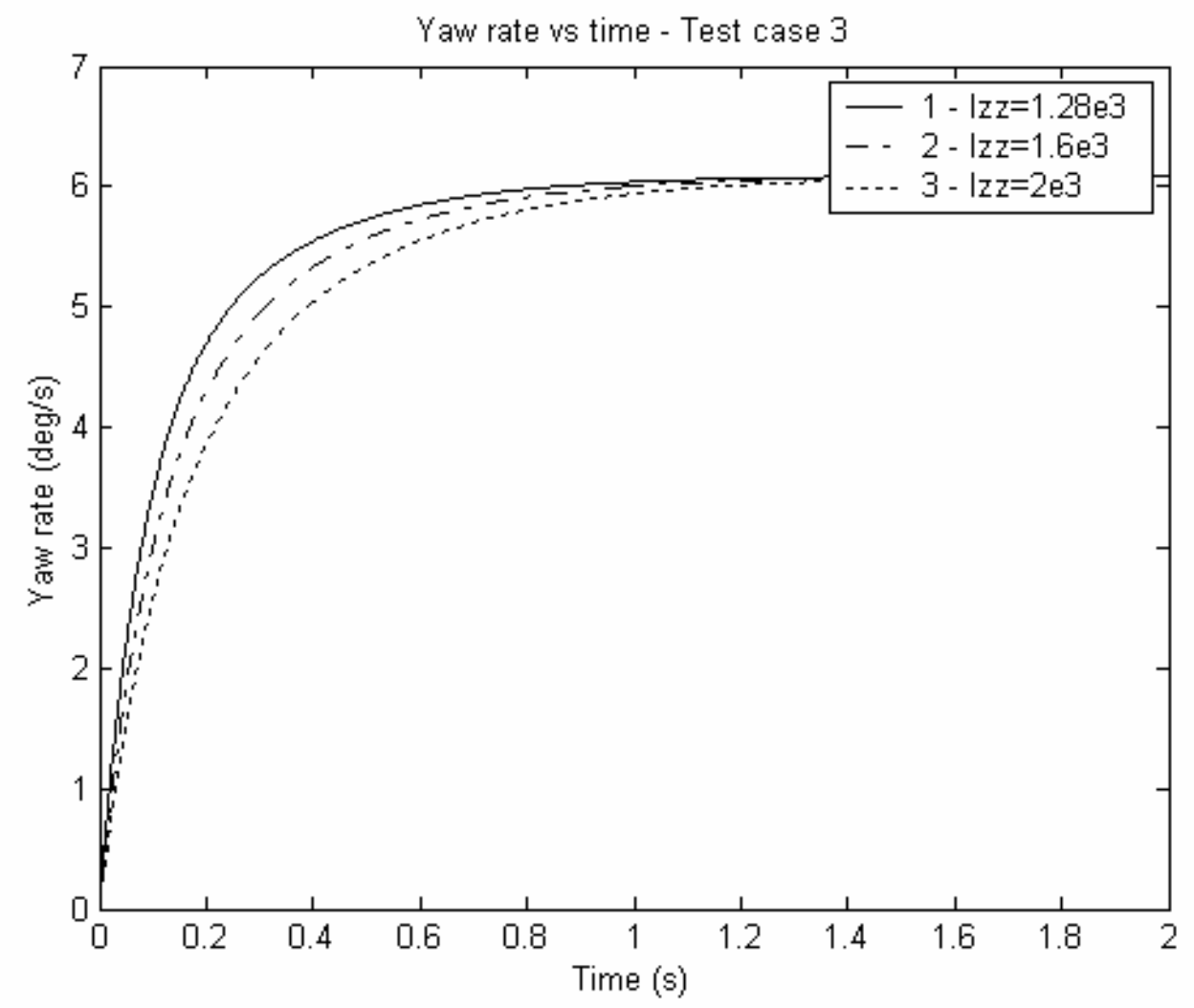

Figure 9 
Roots of the characteristic equation of the yaw response - Test case 3

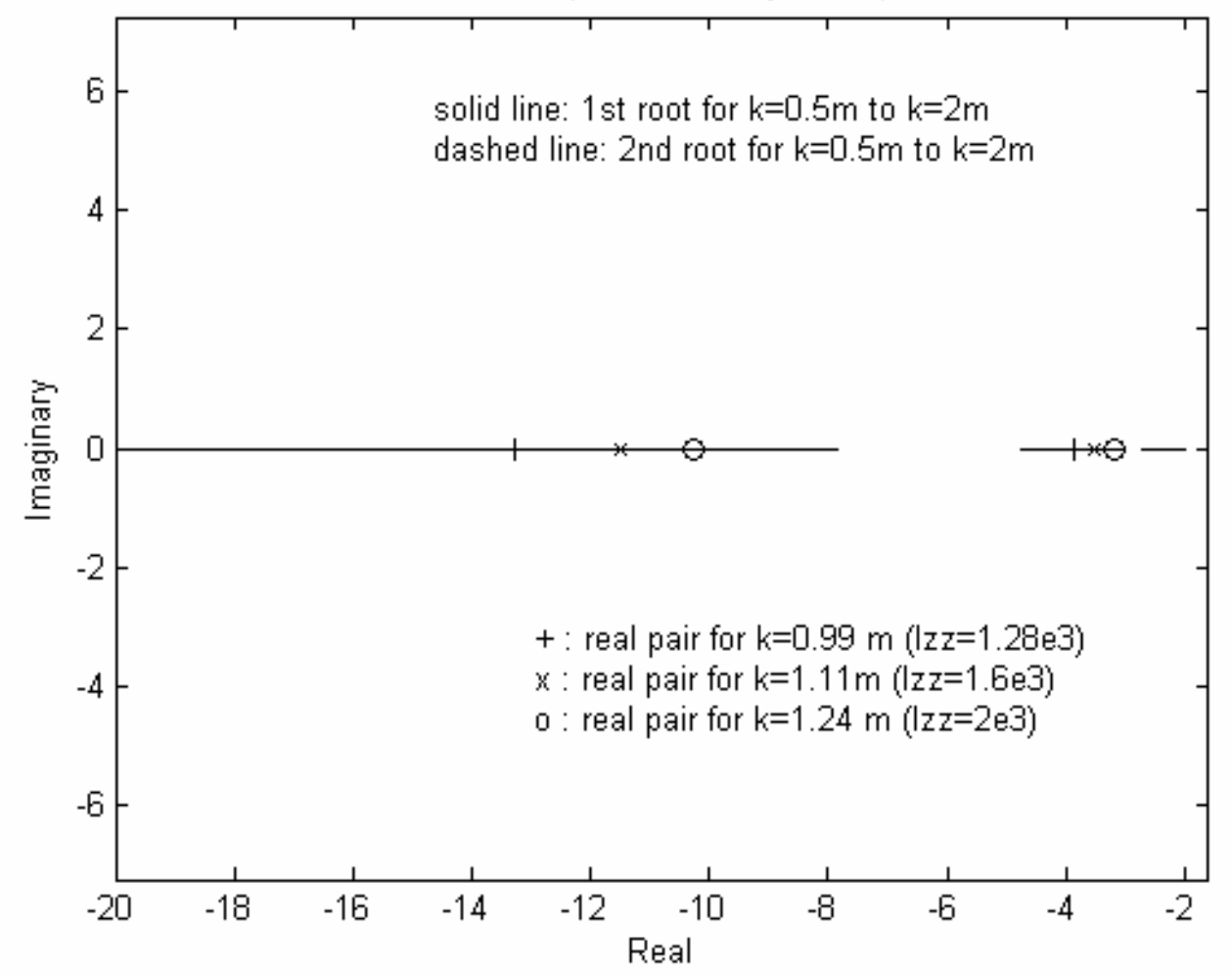

Figure 10 


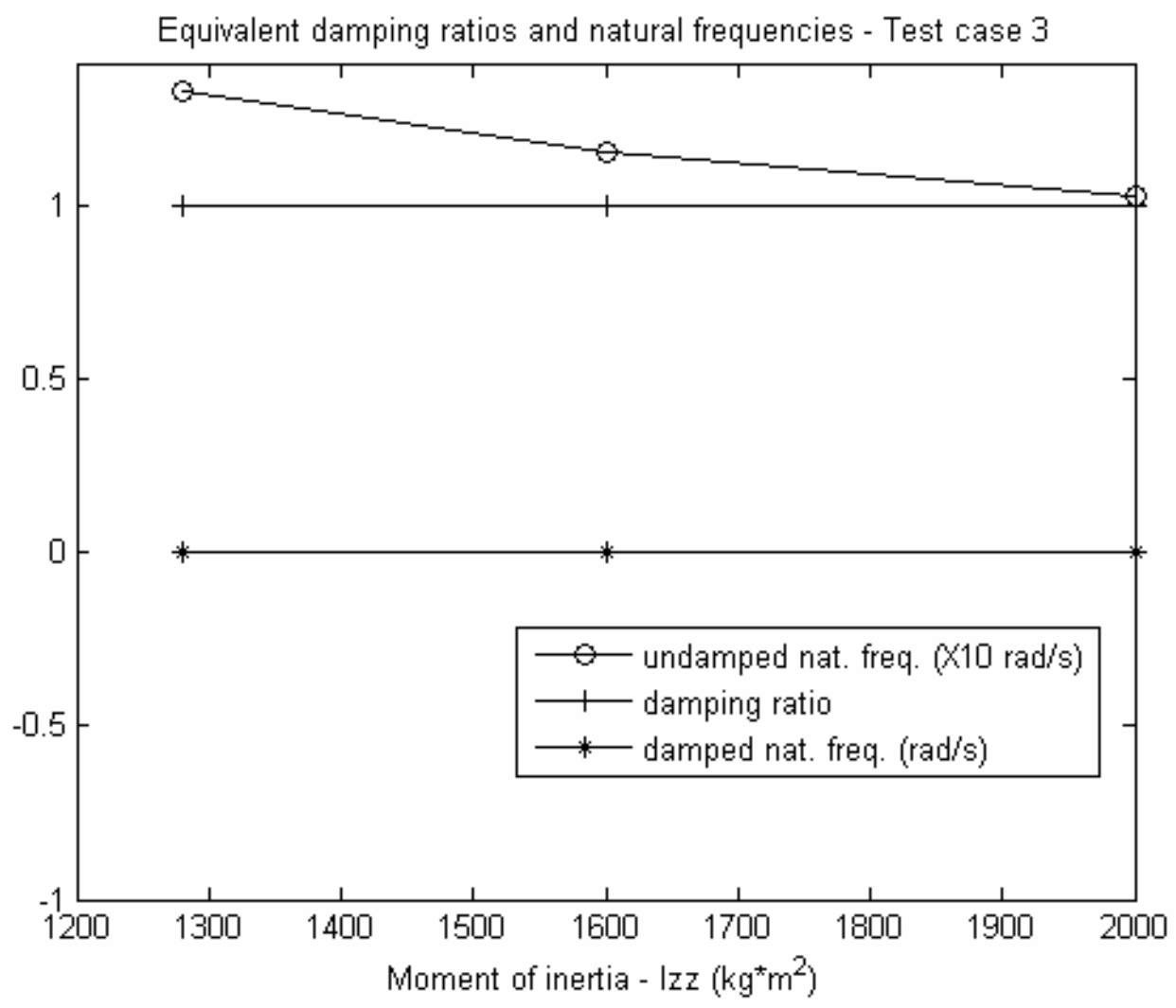

Figure 11 


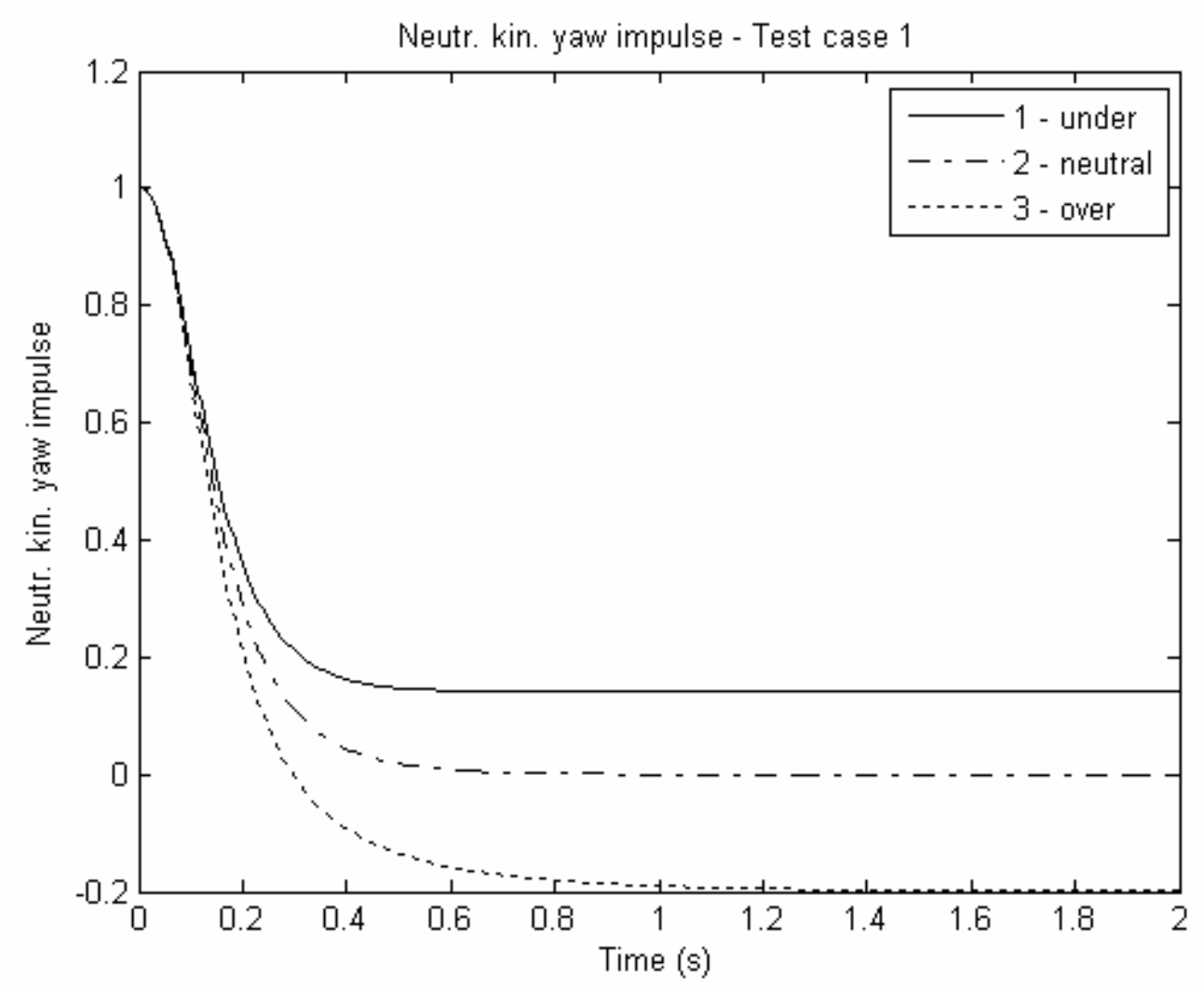

Figure 12 


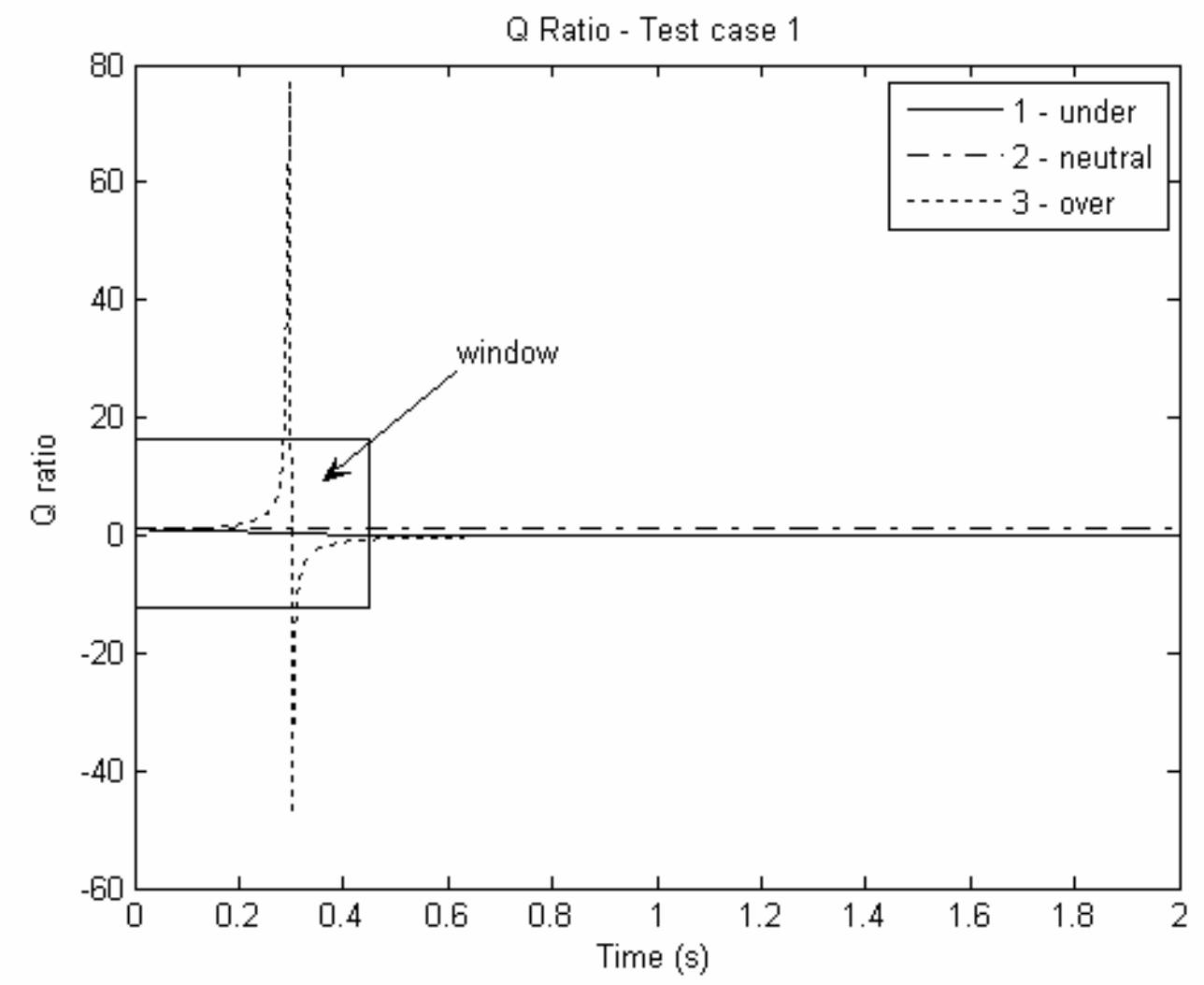

Figure 13 


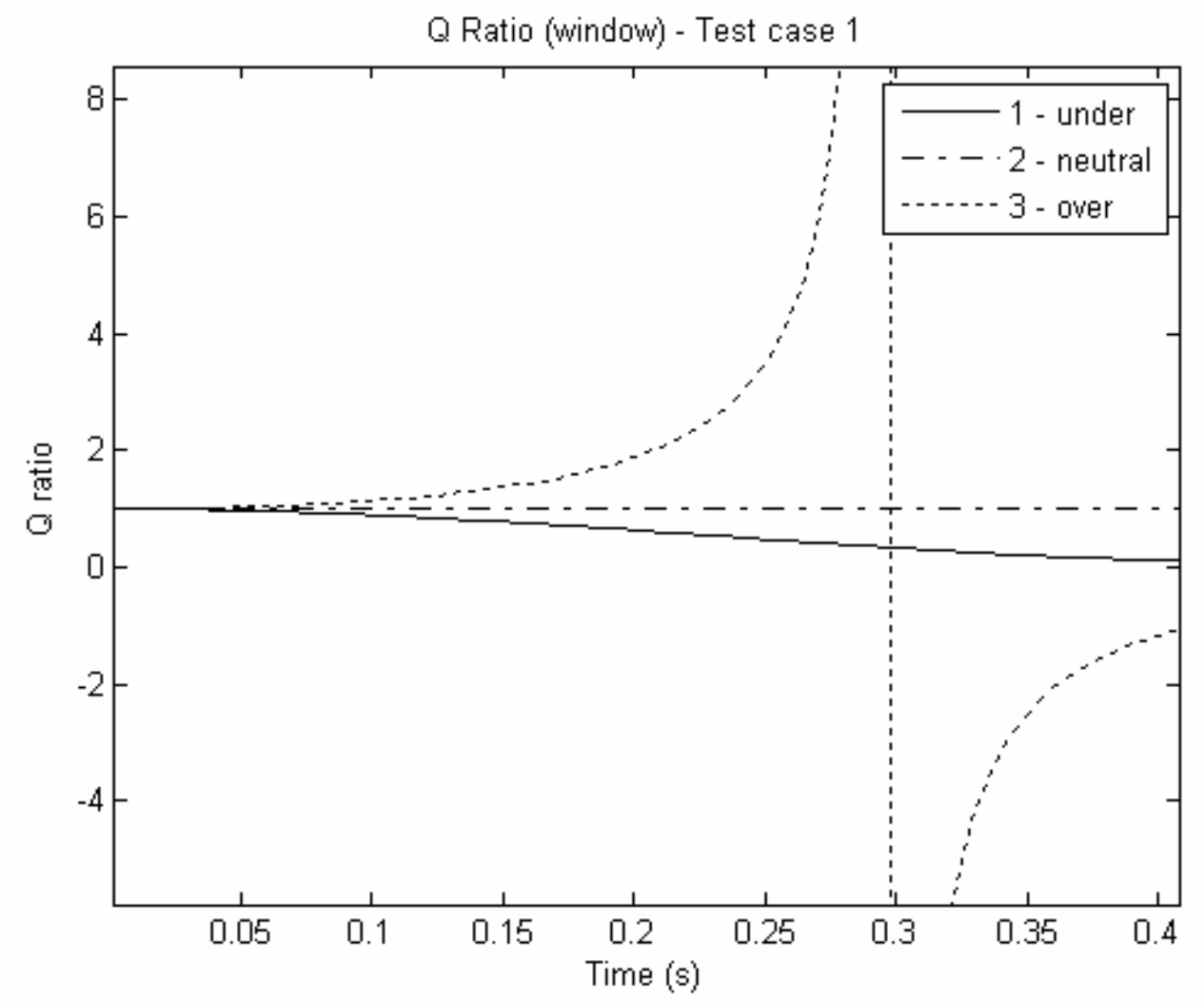

Figure 14 


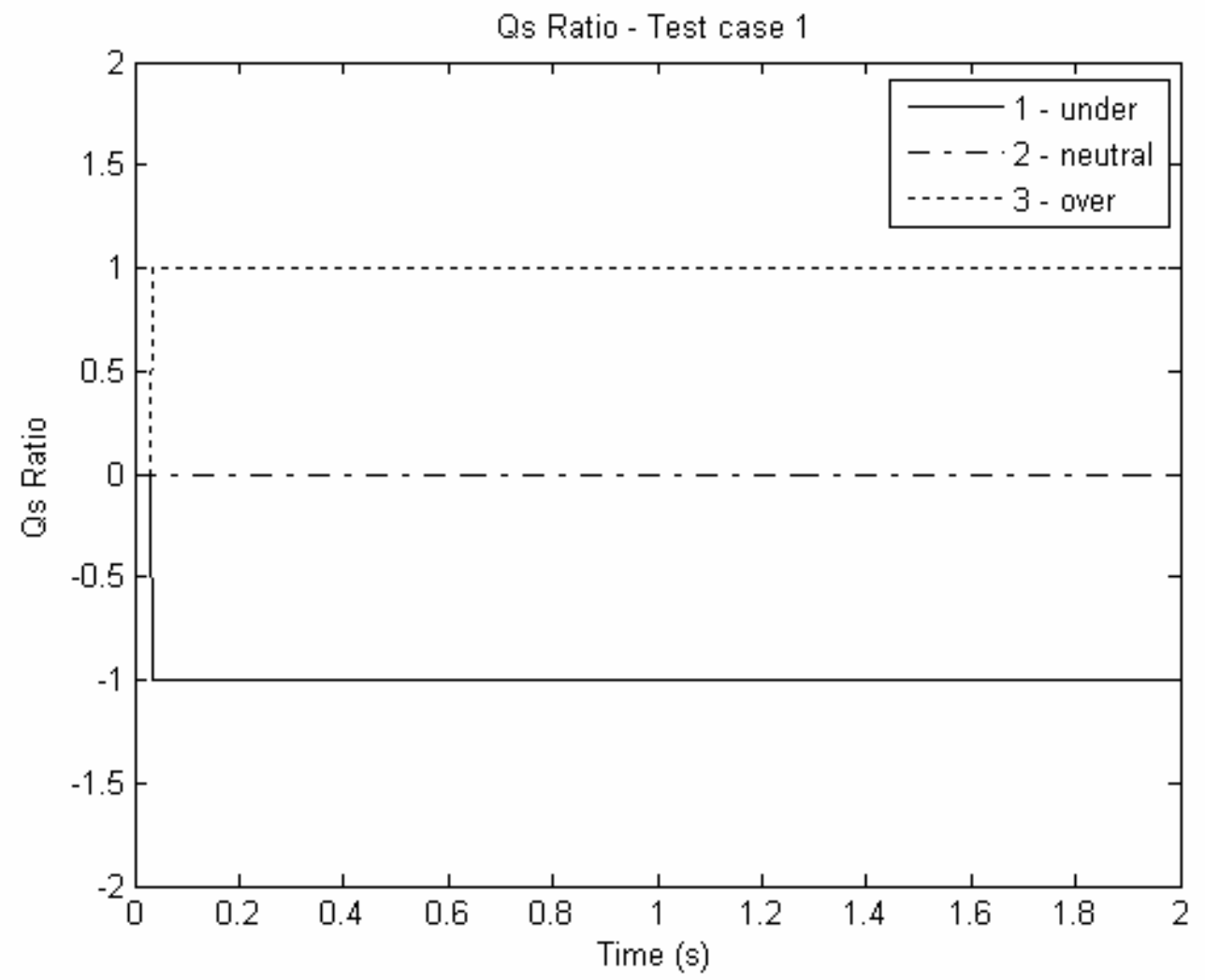

Figure 15 


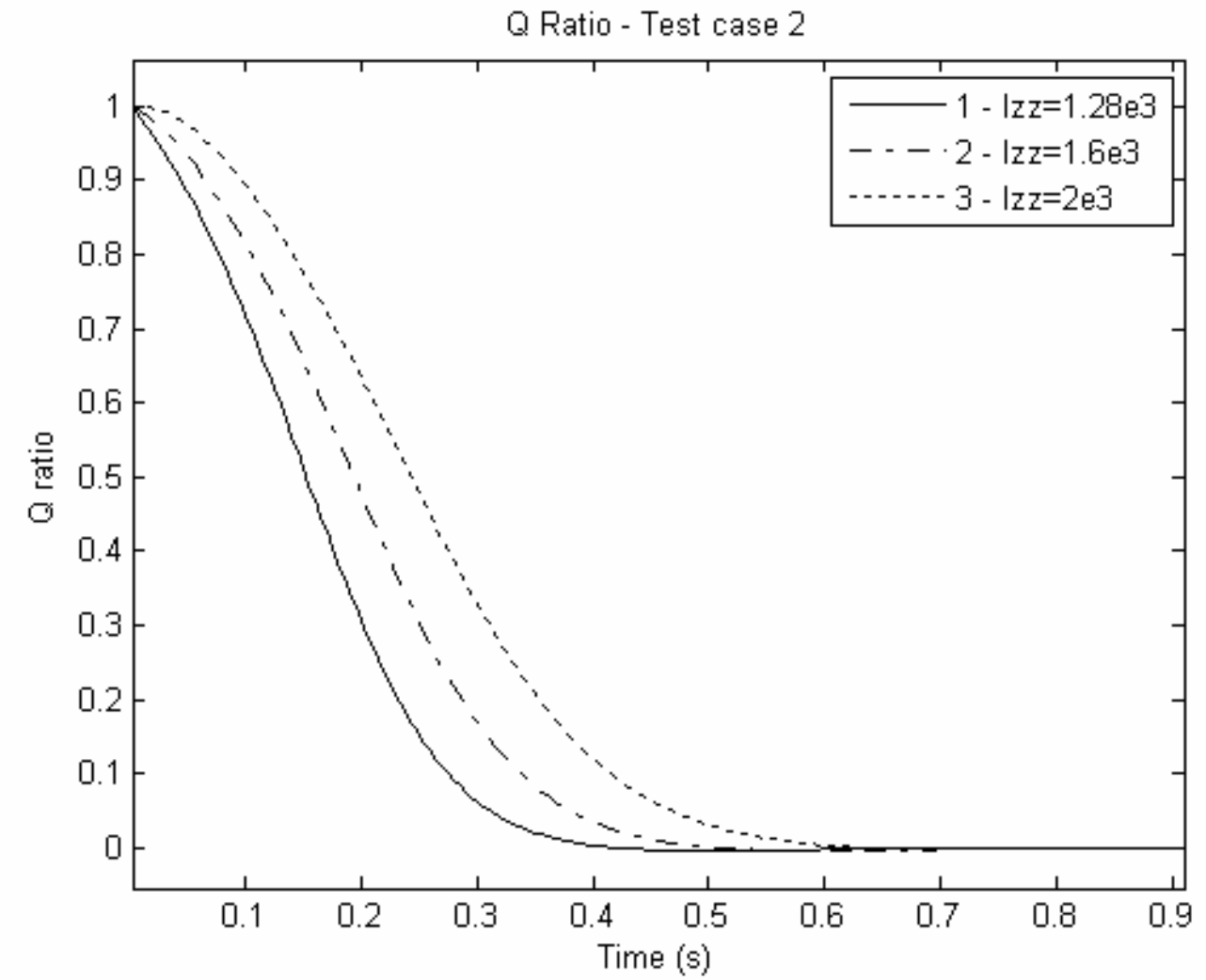

Figure 16 


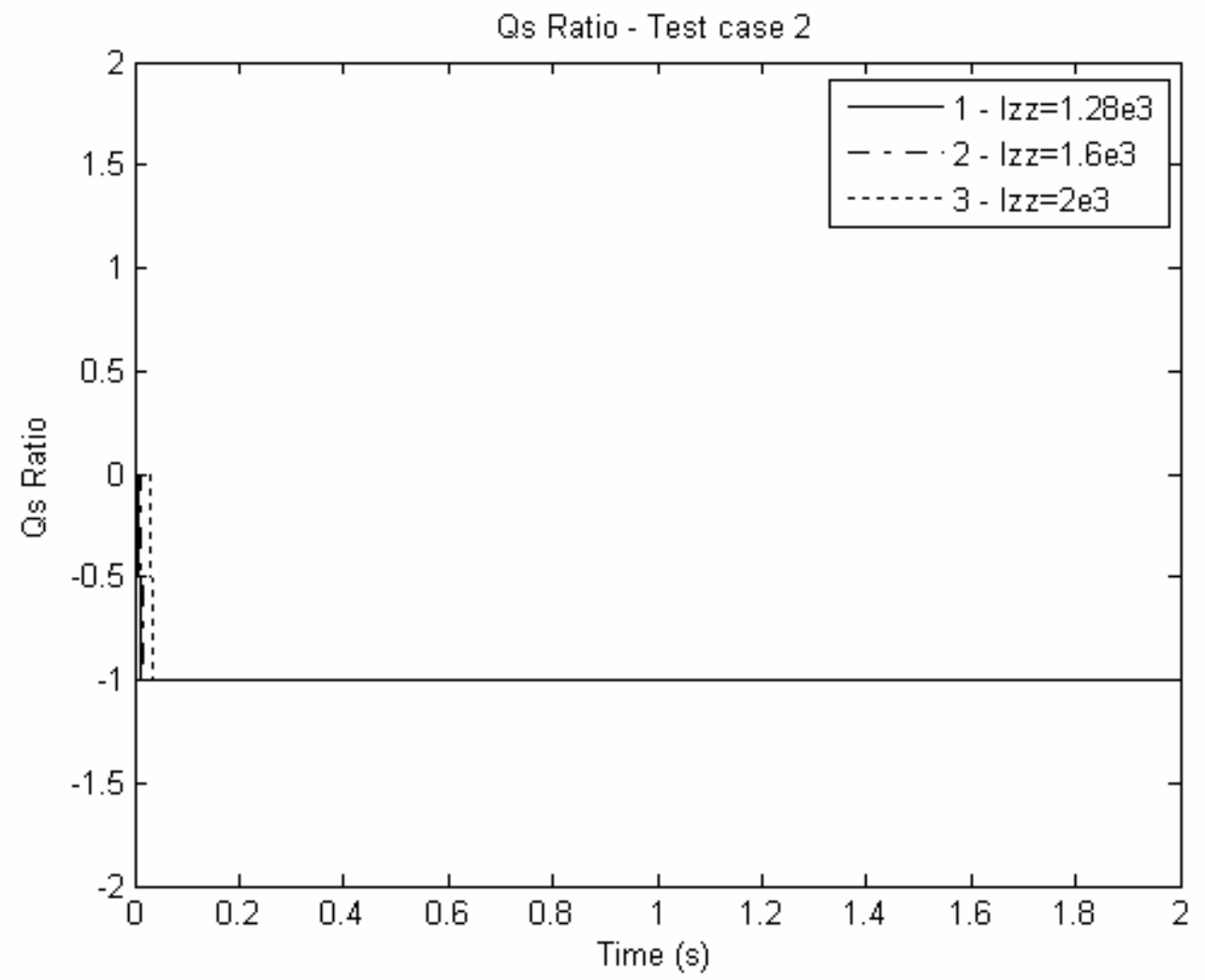

Figure 17 


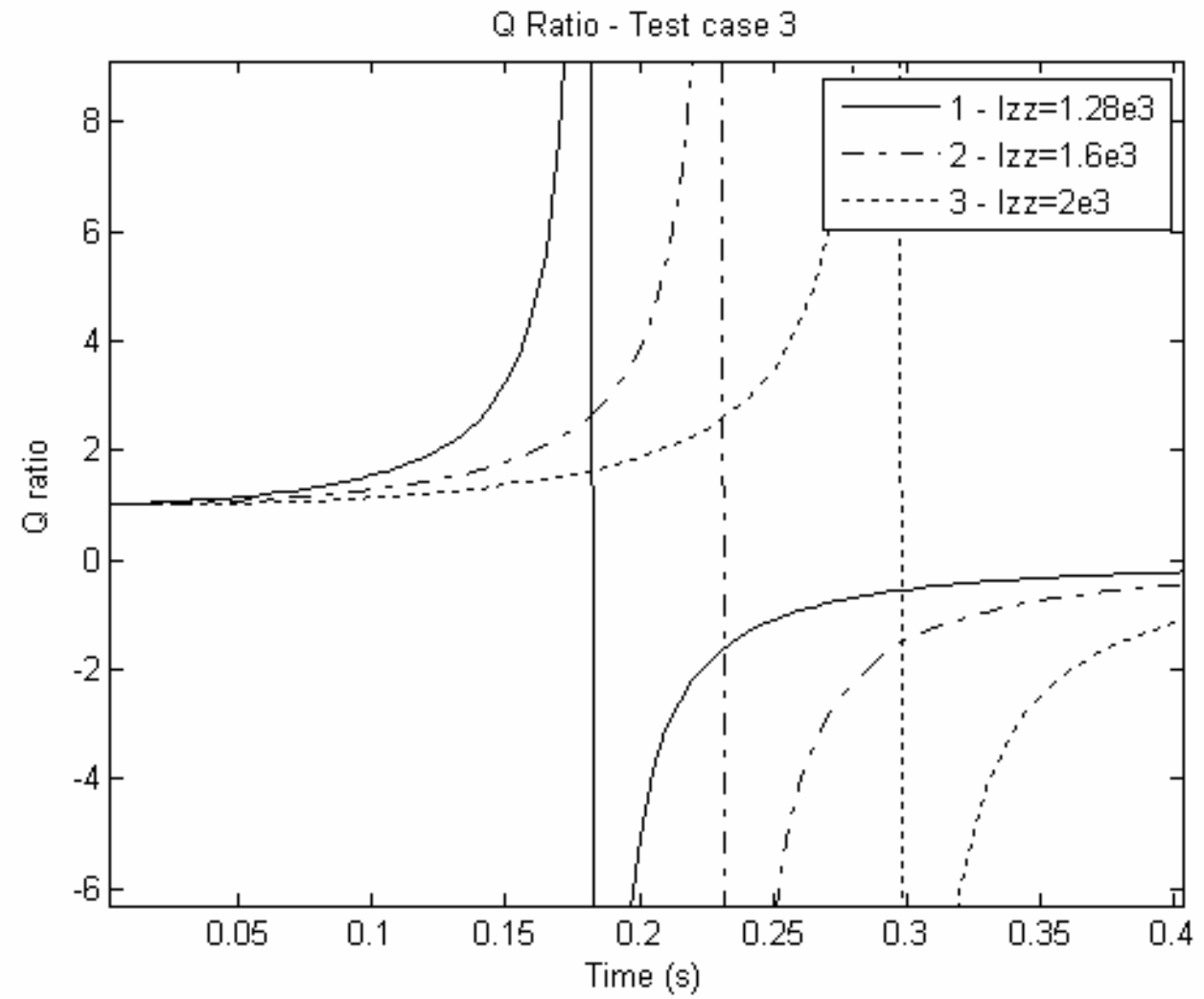

Figure 18 


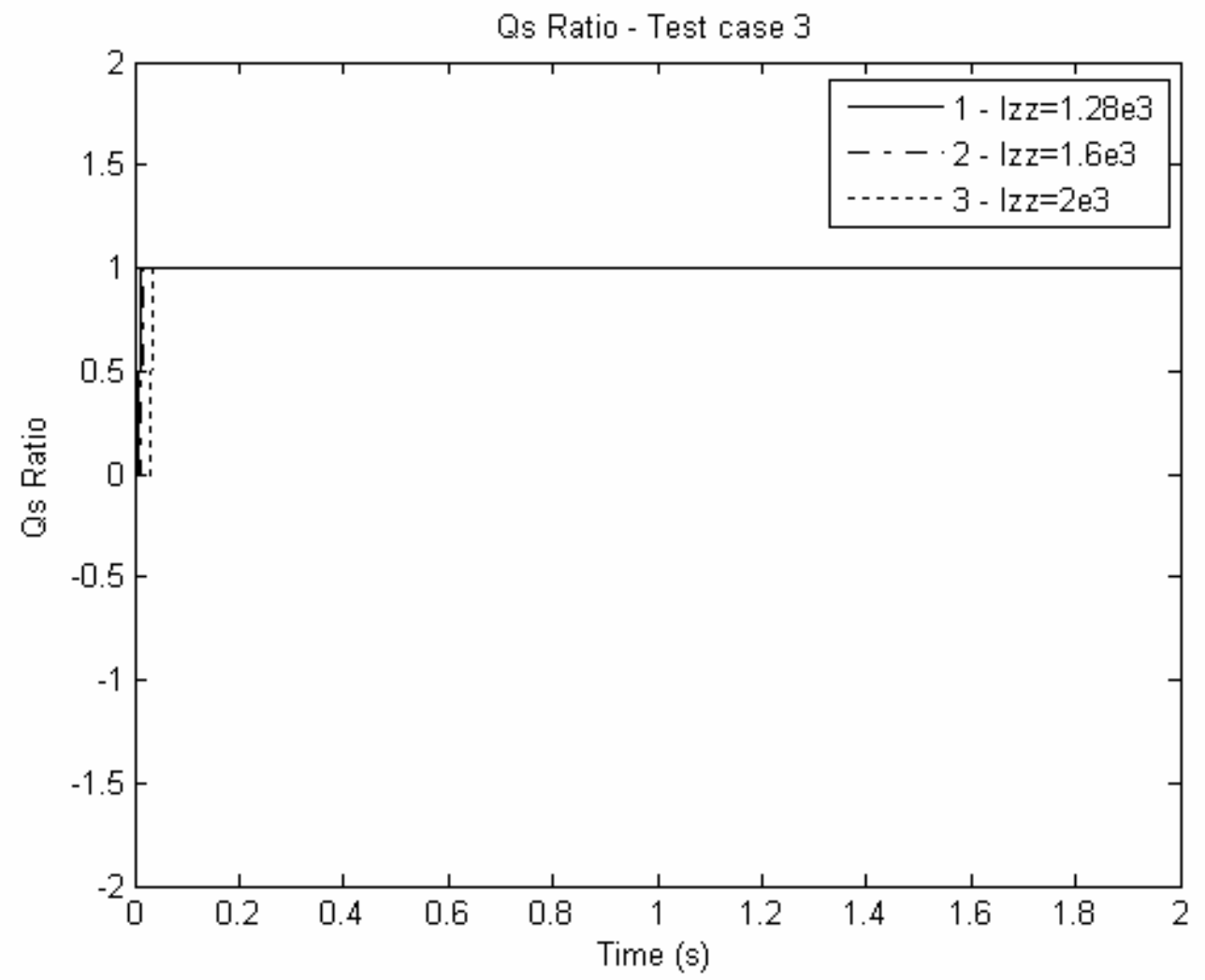

Figure 19 


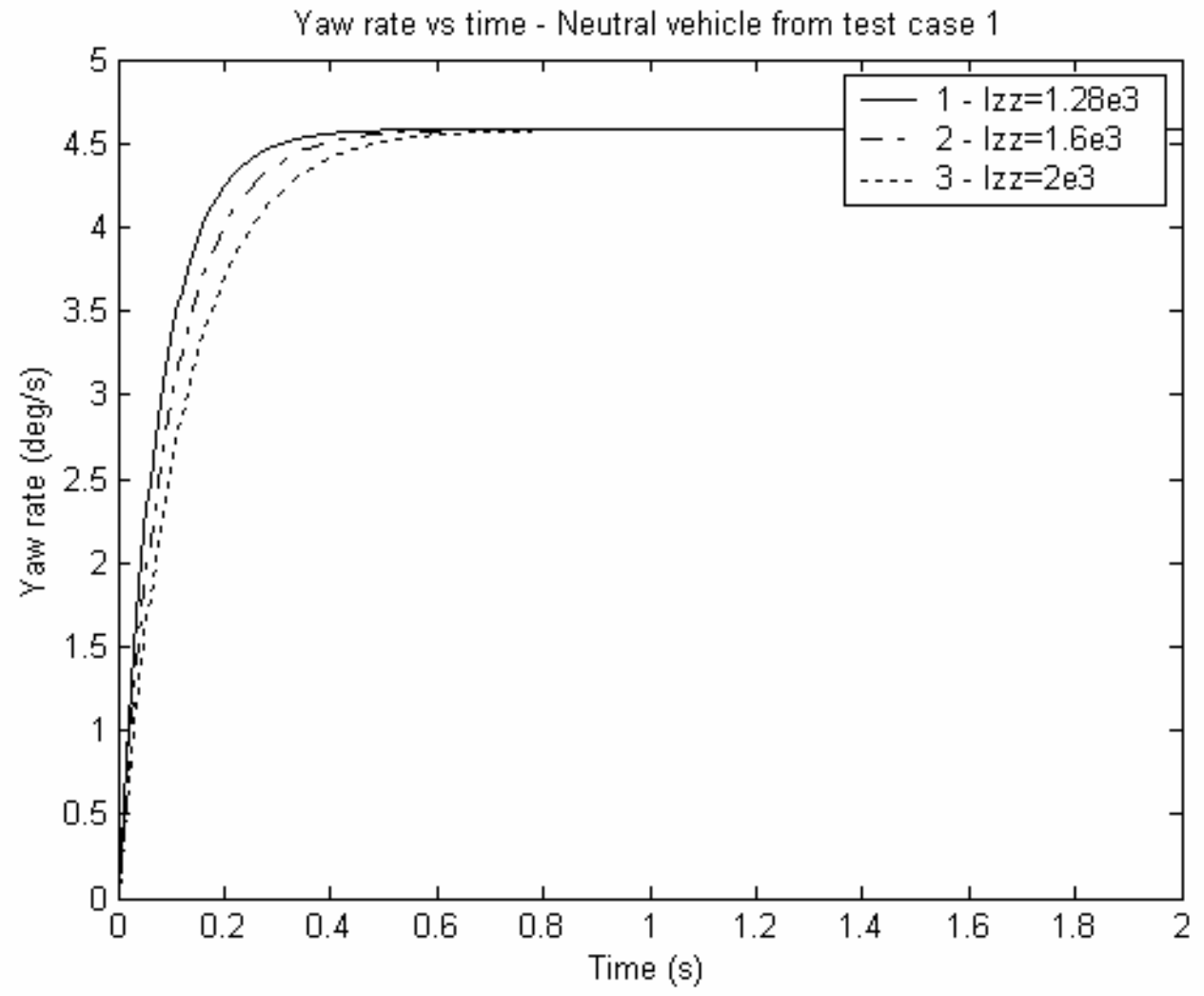

Figure 20 


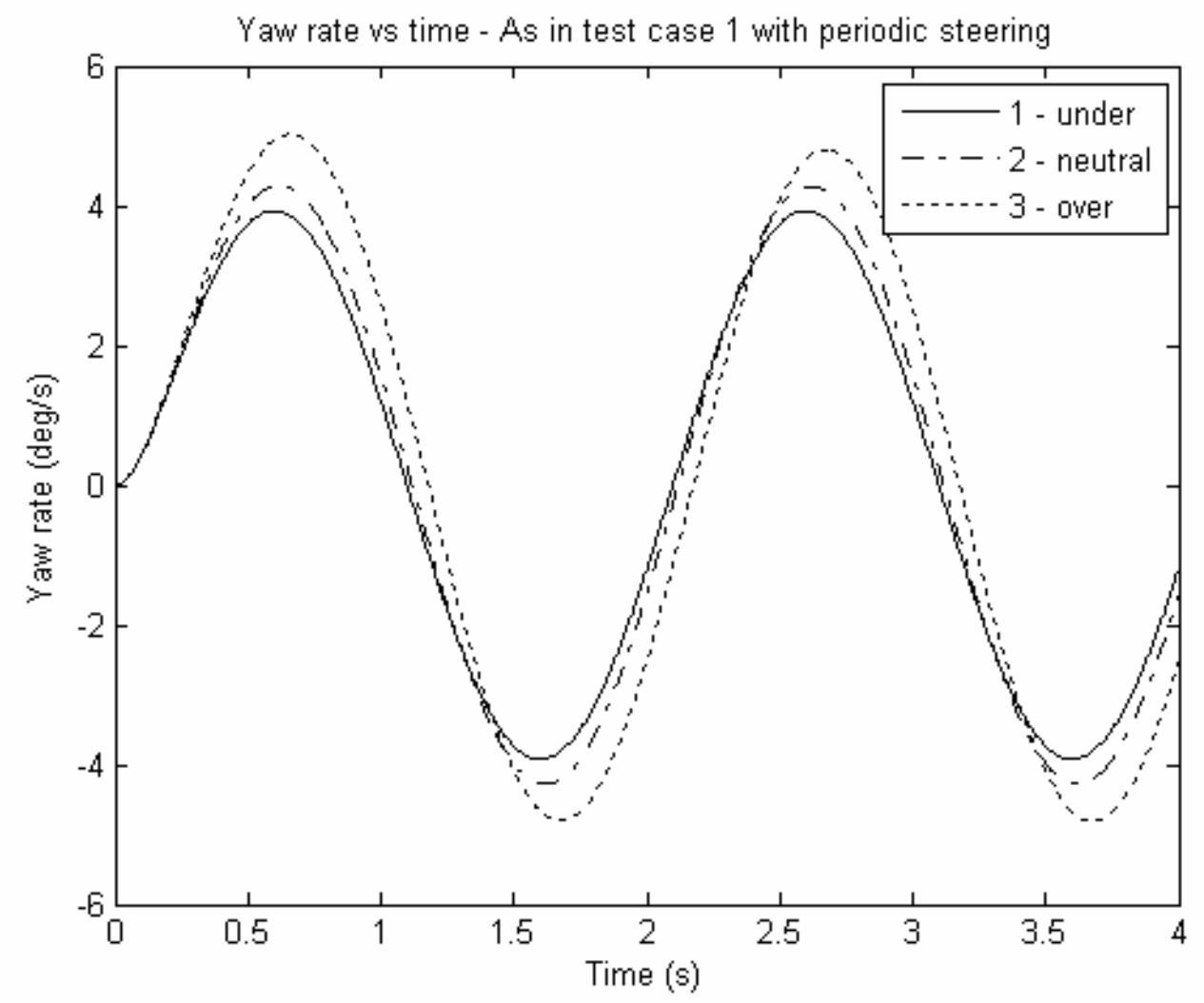

Figure 21 


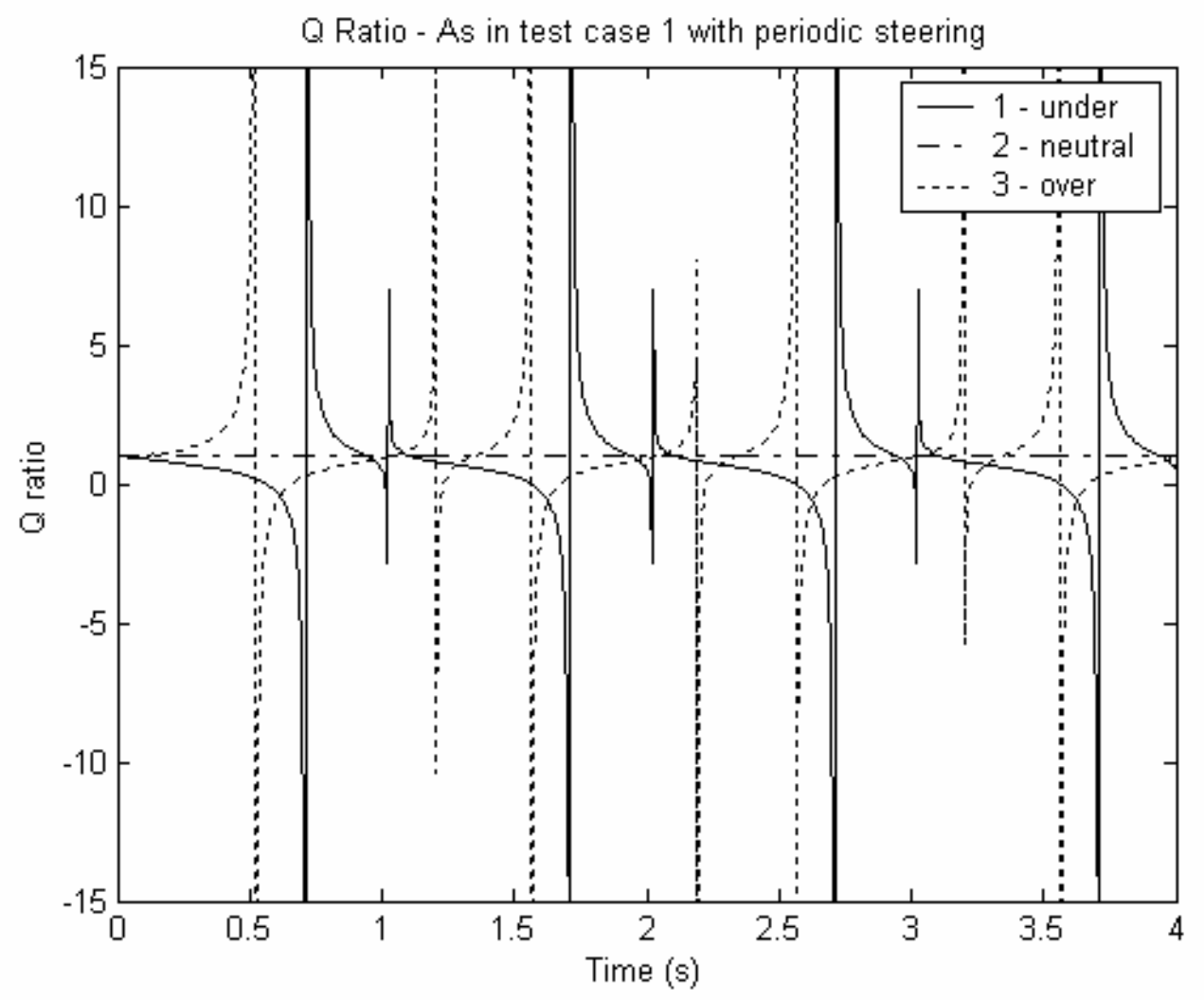

Figure 22 


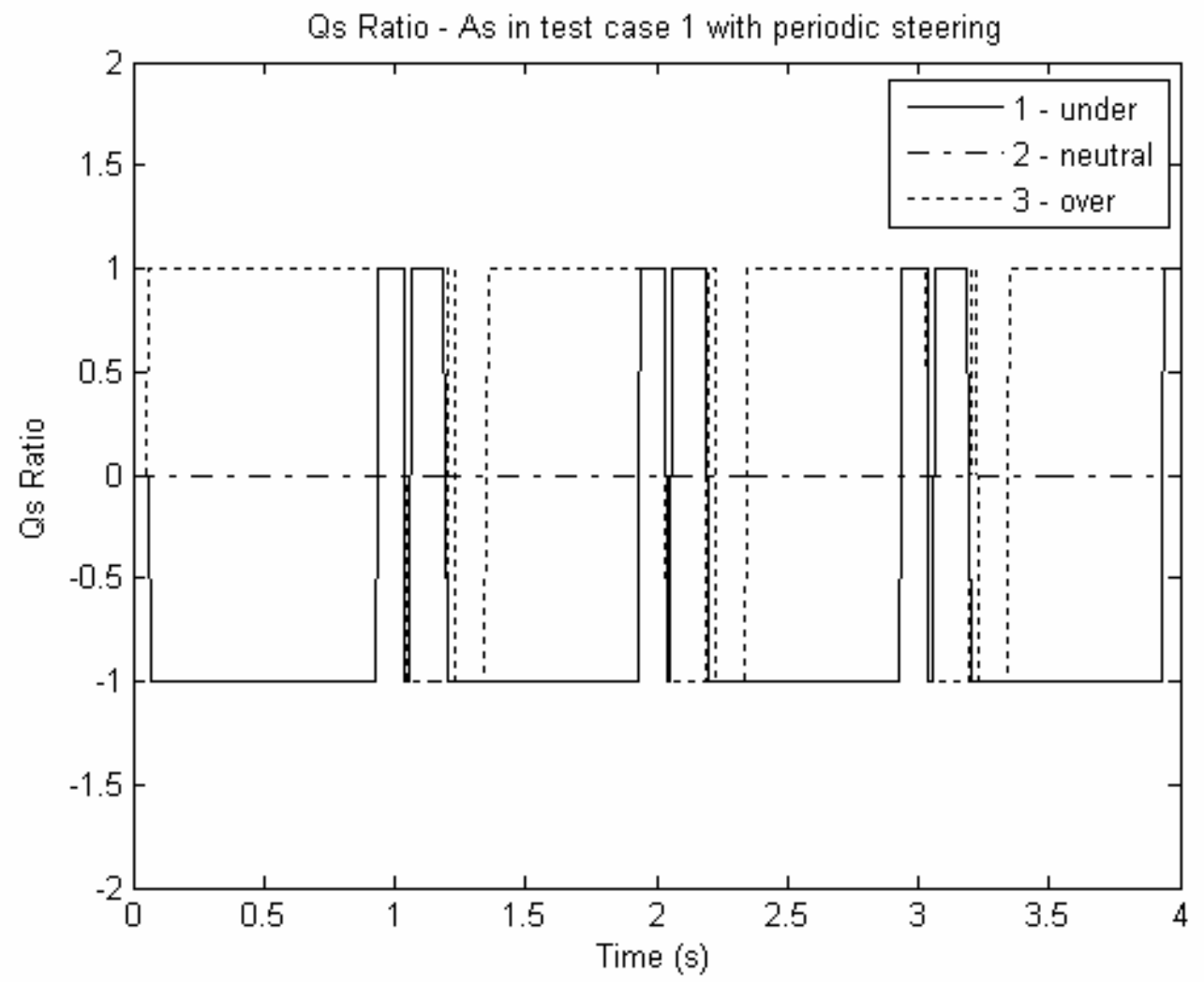

Figure 23 
Table 1

\begin{tabular}{cccccccc}
\hline & $\begin{array}{c}I_{z z} \\
\text { Vehicle No. }\end{array}\left[\mathrm{kg} \cdot \mathrm{m}^{2}\right]$ & $\begin{array}{c}m \\
{[\mathrm{~kg}]}\end{array}$ & $\begin{array}{c}\mathrm{a} \\
{[\mathrm{m}]}\end{array}$ & $\begin{array}{c}\mathrm{b} \\
{[\mathrm{m}]}\end{array}$ & $\begin{array}{c}C_{f} \\
{[\mathrm{~N} / \mathrm{rad}]}\end{array}$ & $\begin{array}{c}C_{r} \\
{[\mathrm{~N} / \mathrm{rad}]}\end{array}$ & $\begin{array}{c}U \\
{[\mathrm{~m} / \mathrm{s}]}\end{array}$ \\
\hline 1 (under) & $2 \mathrm{e} 3$ & $1.3 \mathrm{e} 3$ & 1.0714 & 1.4286 & $6 \mathrm{e} 4$ & $6 \mathrm{e} 4$ & 10 \\
2 (neutral) & $2 \mathrm{e} 3$ & $1.3 \mathrm{e} 3$ & 1.0714 & 1.4286 & $6 \mathrm{e} 4$ & $4.5 \mathrm{e} 4$ & 10 \\
3 (over) & $2 \mathrm{e} 3$ & $1.3 \mathrm{e} 3$ & 1.0714 & 1.4286 & $6 \mathrm{e} 4$ & $3 \mathrm{e} 4$ & 10 \\
\hline
\end{tabular}


Table 2

\begin{tabular}{cccccccc}
\hline & $\begin{array}{c}I_{z z} \\
\text { Vehicle No. }\end{array}\left[\mathrm{kg} \cdot \mathrm{m}^{2}\right]$ & $\begin{array}{c}m \\
{[\mathrm{~kg}]}\end{array}$ & $\begin{array}{c}\mathrm{a} \\
{[\mathrm{m}]}\end{array}$ & $\begin{array}{c}\mathrm{b} \\
{[\mathrm{m}]}\end{array}$ & $\begin{array}{c}C_{f} \\
{[\mathrm{~N} / \mathrm{rad}]}\end{array}$ & $\begin{array}{c}C_{r} \\
{[\mathrm{~N} / \mathrm{rad}]}\end{array}$ & $\begin{array}{c}U \\
{[\mathrm{~m} / \mathrm{s}]}\end{array}$ \\
\hline 1 (under) & $1.28 \mathrm{e} 3$ & $1.3 \mathrm{e} 3$ & 1.0714 & 1.4286 & $6 \mathrm{e} 4$ & $6 \mathrm{e} 4$ & 10 \\
2 (under) & $1.6 \mathrm{e} 3$ & $1.3 \mathrm{e} 3$ & 1.0714 & 1.4286 & $6 \mathrm{e} 4$ & $6 \mathrm{e} 4$ & 10 \\
3 (under) & $2 \mathrm{e} 3$ & $1.3 \mathrm{e} 3$ & 1.0714 & 1.4286 & $6 \mathrm{e} 4$ & $6 \mathrm{e} 4$ & 10 \\
\hline
\end{tabular}


Table 3

\begin{tabular}{cccccccc}
\hline & $\begin{array}{c}I_{z z} \\
\text { Vehicle No. }\end{array}\left[\mathrm{kg} \cdot \mathrm{m}^{2}\right]$ & $\begin{array}{c}m \\
{[\mathrm{~kg}]}\end{array}$ & $\begin{array}{c}\mathrm{a} \\
{[\mathrm{m}]}\end{array}$ & $\begin{array}{c}\mathrm{b} \\
{[\mathrm{m}]}\end{array}$ & $\begin{array}{c}C_{f} \\
{[\mathrm{~N} / \mathrm{rad}]}\end{array}$ & $\begin{array}{c}C_{r} \\
{[\mathrm{~N} / \mathrm{rad}]}\end{array}$ & $\begin{array}{c}U \\
{[\mathrm{~m} / \mathrm{s}]}\end{array}$ \\
\hline 1 (over) & $1.28 \mathrm{e} 3$ & $1.3 \mathrm{e} 3$ & 1.0714 & 1.4286 & $6 \mathrm{e} 4$ & $3 \mathrm{e} 4$ & 10 \\
2 (over) & $1.6 \mathrm{e} 3$ & $1.3 \mathrm{e} 3$ & 1.0714 & 1.4286 & $6 \mathrm{e} 4$ & $3 \mathrm{e} 4$ & 10 \\
3 (over) & $2 \mathrm{e} 3$ & $1.3 \mathrm{e} 3$ & 1.0714 & 1.4286 & $6 \mathrm{e} 4$ & $3 \mathrm{e} 4$ & 10 \\
\hline
\end{tabular}


Table 4

\begin{tabular}{ccc}
\hline & Neutral vs. Under-steer & Neutral vs. Over-steer \\
\hline$I_{z z}$ & error & error \\
\hline$\left[\mathrm{kg} \cdot \mathrm{m}^{2}\right]$ & & 0.971 \\
$1.28 \mathrm{e} 3$ & 0.417 & 0.863 \\
$1.6 \mathrm{e} 3$ & 0.389 & 0.736 \\
\hline $2 \mathrm{e} 3$ & 0.353 & \\
\hline
\end{tabular}

